\title{
Vers le style d'exécution « mainstream » des concertos pour piano de Mozart : l'enregistrement Columbia du Concerto en Sol majeur, K. 453, par Ernst von Dohnányi ${ }^{1}$
}

\author{
Adriano GiaRdinA \\ Université de Fribourg - Faculté des lettres et des sciences humaines \\ Domaine Histoire de l'art, Archéologie et Musicologie \\ Avenue de l'Europe 20, Bureau 2031, CH-1700 Fribourg, Suisse \\ E-mail : adriano.giardina@unifr.ch
}

(Reçu : mars 2017 ; accepté : mai 2017)

\begin{abstract}
The gramophone recording of Mozart's Piano Concerto in G major, K. 453, featuring Ernst von Dohnányi as soloist and conductor of the Budapest Philharmonic Orchestra, made in 1928 for the Columbia Company, is important in many respects. The Hungarian pianist and composer made little more than a handful of gramophone recordings until the late 1940s. This performance is also the first audio recording ever to be published that contained a Mozart piano concerto (some piano rolls with concertos or extracts did exist beforehand). From the beginning of his career, Dohnányi had been one of the keenest promoters of the Austrian composer's piano pieces. In the Columbia recording, the performing style of Dohnányi and his orchestra is characteristic of its time, notably because it chooses to use a flexible tempo. In addition, the soloist makes use of rubato and chord dislocation. Nonetheless, the performers are also playing in an intimate conversational tone and they emphasize Mozart's structural clarity. The execution of themes by the pianist is both poetic and restrained. These traits will define the "mainstream" performing style of Mozart's piano concertos over most of the twentieth century. An implicit aesthetic standard comes into force in the critical reviews of the Columbia records: Mozart's piano concertos require lightness and gentleness from the soloist. The elements given prominence to the recording and in the reviews also appear in contemporary musicological literature and in texts on music. Recordings of two additional Mozart piano concertos (K. 271 and K. 503), played live by Dohnányi in the 1950 s, display a broadly similar performing style. Over the ten years that followed the Columbia recording, the majority of Mozart's "great" piano concertos were published on records. This newly found popular interest is connected with a positive re-evaluation of this group of Mozart's works.
\end{abstract}

Keywords: Mozart, Piano Concerto in G major (K. 453), Piano Concerto in E-flat major (K. 271), Ernst von Dohnányi, performance history

1. Cet article est la version écrite de la communication que j’ai prononcée lors du Dohnányi Day 2017, à Budapest. Je remercie Maxime Goergen pour sa relecture de la version anglaise de mon abstract. 
En juin 1928, Ernst von Dohnányi est en tournée à Londres avec l'Orchestre Philharmonique de Budapest, dont il est le chef titulaire. ${ }^{2}$ Le public londonien connait déjà bien le pianiste et compositeur hongrois, mais c'est la première fois que Dohnányi dirige l'orchestre, composé de cent dix musiciens, dans la capitale anglaise. Le 15 juin, lors d'un concert, le pianiste joue et dirige depuis son instrument le Concerto pour piano et orchestre $n^{\circ} 17$ en Sol majeur, K. 453, de Mozart. Dohnányi et l'orchestre profitent de ce séjour pour enregistrer l'œuvre chez Columbia. ${ }^{3}$ En outre, le musicien est alors désigné membre d'honneur de la Royal Academy of Music.

Cette captation est importante à plusieurs égards. Comme le pianiste, considéré comme un des plus importants dès ses débuts internationaux au tournant du $\mathrm{XX}^{\mathrm{e}}$ siècle, a laissé relativement peu d'enregistrements, chacun d'eux mérite qu'on s'y attarde. Dohnányi a réalisé quelques rouleaux pour piano mécanique au début du siècle, avec de la musique de Haydn, Beethoven, Schubert, Chopin, Liszt, Brahms et Delibes, ainsi qu'avec quelques-unes de ses propres œuvres. ${ }^{4}$ Durant les années 1920 à 1950, il n'enregistre qu'une dizaine de disques, consacrés, mis à part le concerto de Mozart qui va nous occuper ici, en bonne partie à ses propres pièces pour piano. ${ }^{5}$ Dans ses dernières années, qu'il passe aux Etats-Unis, et alors qu'il est pianiste et compositeur en résidence à la Florida State University, Dohnányi se produit souvent lors des concerts, qui ont été enregistrés. Ces captations sont aujourd'hui conservées dans la Collection Dohnányi à l'Institut de musicologie de l'Académie hongroise des sciences. ${ }^{6}$ Le fonds préserve des enregistrements de musique pour piano, de musique de chambre, de musique d'orchestre et même de musique vocale avec orchestre. ${ }^{7}$ Pour ce qui est de la musique pour ou avec piano

2. Voir Ilona von Dohnányi, Ernst von Dohnányi. A Song of Life, éd. James A. Grimes (Bloomington, Indianapolis : Indiana University Press, 2002), 92. ; William Waterhouse, «Dohnányi and Great Britain », in Perspectives on Ernst von Dohnányi, éd. James A. Grymes (Lanham, Maryland-Toronto-Oxford : The Scarecrow Press, 2005), 62.

3. Quatre disques, Columbia L 2215-2218 (matrices : WAX 3790-3797). La captation a été republiée en CD entre autre par le label Schwann en 1990 (Ernst von Dohnanyi spielt und dirigiert, 1 CD, Koch Schwann Musica Mundi, CD 311 136, 1990). Le transfert digital a été réalisé par Mark Obert-Thorn. Mes analyses sont basées sur ce repiquage.

4. Voir János Mácsai, « Megjegyzések Dohnányi Ernő gépzongora-felvételeinek jegyzékéhez », Holmi 6/5 (mai 1994), 726-732 ; James A. Grymes, Ernst von Dohnányi. A Bio-Bibliography (Westport, Connecticut-Londres: Greenwood Press, 2001) = Bio-Bibliographies in Music, 86, 71-82. ; Deborah Kiszely-Papp, «Dohnányi Ernő müvei és előadóművészi munkássága hangfelvételeken », Dohnányi Évkönyv 2002, éd. Márta Sz. Farkas (Budapest : MTA Zenetudományi Intézet, 2002), 161-190.

5. Voir Grymes, Dohnányi. A Bio-Bibliography, 71-82 ; Deborah Kiszely-Papp, « Dohnányi Ernő művei és előadóművészi munkássága hangfelvételeken ».

6. Voir Veronika Kusz, «From Budapest to Florida - And Back: The Journey Taken by Ernst von Dohnányi's American Legacy », Notes: Quarterly Journal of the Music Library Association 73/4 (2017), $658-672$.

7. Un catalogue figure dans Veronika Kusz, Dohnányi amerikai évei (Budapest : Rózsavölgyi és Társa, 2015), 328-345. Quelques-unes de ces captations viennent d'être republiées en CD : The Last Romantic. Dohnányi's American concert recordings, 2 CDs, éd. Veronika Kusz (Budapest : Rózsavölgyi és Társa, RÉTCD 089, 2017). Une captation de 1952 des deux premiers mouvements de la Sonate pour piano et violon $n^{\circ} 32$ en Si bémol majeur, K. 454 y est incluse. Dohnányi a ici Albert Spalding pour partenaire. Un enregistrement 
de Mozart, nous avons des enregistrements de la Sonate pour piano $n^{\circ} 11$ en La majeur, K. 331, de la Fantaisie pour piano en do mineur, K. 475, de la Sonate pour deux pianos en Ré majeur, K. 448, que Donányi interprète avec Ede Kilényi. Deux concertos pour piano et orchestre figurent également dans le lot : le $n^{\circ} 9$ en Mi bémol majeur, K. 271, et le $n^{\circ} 25$ en Do majeur, K. 503. Dohnányi est accompagné par un orchestre d'étudiants, le Ohio University Orchestra, dirigé par Karl Arhendt. Sont en outre conservés des enregistrements de sonates pour piano et violon. ${ }^{8}$

L'enregistrement du Concerto en Sol de 1928 compte également du point de vue de l'histoire de l'interprétation de la musique pour piano de Mozart, dans la mesure où c'est le premier concerto véritablement accompagné par un orchestre dont on conserve une captation. En effet, quelques interprètes ont enregistré des concertos pour piano ou des extraits sous forme de rouleaux pour piano. Bien entendu, il s'agit là d'arrangements dans lesquels les pianistes jouent seuls les parties dévolues au soliste et à l'orchestre. Le Tableau 1 répertorie ces rouleaux. ${ }^{9} \mathrm{Si}$ la plupart de ces enregistrements datent très certainement d'avant 1928, quelques uns d'entre eux ont pu être réalisés cette année-là ou quelque peu après, mais il est impossible d'en dire plus dans l'état actuel de la recherche. ${ }^{10}$ Pour beaucoup d'entre eux, nous n'avons conservé aucun exemplaire des rouleaux. On peut noter une préférence des interprètes pour le second mouvement du Concerto $n^{\circ} 26$ en Ré majeur, K. 537. Le Concerto en Sol n'apparaît pas ici.

Ce n'est pas tout. Cet enregistrement présente des aspects étonnants si on les rapporte à la réception de Dohnányi après la Seconde Guerre Mondiale. Voici un extrait du bref développement que le critique américain Harold C. Schonberg consacre au pianiste dans son livre The Great Pianists from Mozart to the Present, dont la première édition remonte à 1963 : « His [Dohnányi's] playing had power and propulsion, and extraordinary finesse. Naturally he was a romantic pianist (his recording of the Mozart $\mathrm{G}$ major Concerto contains everything that is considered bad style today), just as his own compositions are in the romantic

de la Sonate pour piano $n^{\circ} 11$ en La majeur, K. 331, apparaît sur le CD Dohnányi at the piano. 1955, 1956, 1959, (Budapest : Hungaroton, HCD 12085, 1993). Mes remerciements vont à Veronika Kusz et László Stachó pour leur gentillesse et leur aide concernant ces enregistrements.

8. Les sonates $n^{\circ} 18$ en Sol majeur, K. 301, $n^{\circ} 21$ en mi mineur, K. 304, $n^{\circ} 26$ en Si bémol majeur, K. 378 et $n^{\circ} 32$ en Si bémol majeur, K. 454, avec Ede Zathureczky; la Sonate $n^{\circ} 32$ en Si bémol majeur, K. 454, avec Albert Spalding.

9. Cette liste a été établie à partir de Gerhard Dangel et Hans-W. Schmitz, Gesamtkatalog der europäischen Aufnahmen 1904-1932 für das Welte-Mignon Reproduktionspiano (Stuttgart : Schmitz, 2006) ; Larry Sitsky, The Classical Reproducing Piano Roll. A Catalogue-Index, 2 vols. (New York : Greenwood Press, 1990) = Music Reference Collection, 23.

10. Seule la marque Welte-Mignon a pour l'heure fait l'objet d'investigations poussées. Voir par exemple Aus Freiburg in die Welt: 100 Jahre Welte-Mignon - Automatische Musikinstrumente, éd. Gerhard Dangel (Freiburg im Breisgau : Augustinermuseum, 2005). 
TABleau 1 Rouleaux de piano préservant des concertos pour piano de Mozart (ou des extraits)

\begin{tabular}{|c|c|c|c|}
\hline $\begin{array}{l}\text { Année } \\
\text { d'enregis- } \\
\text { trement }\end{array}$ & Euvre & Pianiste & Compagnie \\
\hline 1905 & $\begin{array}{l}\text { Concerto } n^{\circ} 3 \text { en Ré majeur, K. } 40, \text { ou } \\
\text { Concerto } n^{\circ} 5 \text { en Ré majeur, K.175, } \\
\text { Premier mouvement }\end{array}$ & $\begin{array}{l}\text { Adam-Benard } \\
\text { Eugenie }\end{array}$ & Welte-Mignon \\
\hline 1905 & $\begin{array}{l}\text { Concerto } n^{\circ} 26 \text { en Ré majeur, K. } 537 \text {, } \\
\text { Deuxième mouvement }\end{array}$ & $\begin{array}{l}\text { Adam-Benard } \\
\text { Eugenie }\end{array}$ & Welte-Mignon \\
\hline 1905 & $\begin{array}{l}\text { Concerto } n^{\circ} 26 \text { en Ré majeur, K. } 537 \text {, } \\
\text { Deuxième mouvement }\end{array}$ & Reinecke Carl & Welte-Mignon \\
\hline 1912 & Concerto $n^{\circ} 23$ en La majeur, K. 488 & Weiland Fanny & Welte-Mignon \\
\hline 1923 & $\begin{array}{l}\text { Concerto } n^{\circ} 22 \text { en Mi bémol majeur, K. } 482 \text {, } \\
\text { Deuxième mouvement }\end{array}$ & Fischer Edwin & Welte-Mignon \\
\hline$?$ & $\begin{array}{l}\text { Concerto } n^{\circ} 26 \text { en Ré majeur, K. } 537 \text {, } \\
\text { Deuxième mouvement }\end{array}$ & Reinecke Carl & Ampico \\
\hline$?$ & $\begin{array}{l}\text { Concerto } n^{\circ} 26 \text { en Ré majeur, K. } 537 \text {, } \\
\text { Deuxième mouvement }\end{array}$ & Silber Sidney & Ampico \\
\hline$?$ & $\begin{array}{l}\text { Concerto } n^{\circ} 20 \text { en ré mineur, K. } 466 \text {, } \\
\text { Deuxième mouvement }\end{array}$ & $\begin{array}{l}\text { Mayer-Mahr } \\
\text { Moritz }\end{array}$ & Duca \\
\hline$?$ & $\begin{array}{l}\text { Concerto } n^{\circ} 26 \text { en Ré majeur, K. } 537 \text {, } \\
\text { Deuxième mouvement }\end{array}$ & Grunfeld Alfred & Duca \\
\hline$?$ & $\begin{array}{l}\text { Concerto } n^{\circ} 26 \text { en Ré majeur, K. } 537 \text {, } \\
\text { Deuxième mouvement }\end{array}$ & $\begin{array}{l}\text { Muller-Reuter } \\
\text { Theodor }\end{array}$ & Duca \\
\hline$?$ & $\begin{array}{l}\text { Concerto } n^{\circ} 12 \text { en La majeur, K. } 414 \text {, ou } \\
\text { Concerto } n^{\circ} 23 \text { en La majeur, K. } 488\end{array}$ & Casadesus Robert & Pleyela \\
\hline$?$ & Concerto $n^{\circ} 9$ en Mi bémol majeur, K. 271 & Pugno Raoul & Triphonola \\
\hline$?$ & Concerto $n^{\circ} 9$ en Mi bémol majeur, K. 271 & Rehberg Willy & Triphonola \\
\hline$?$ & $\begin{array}{l}\text { Concerto } n^{\circ} 20 \text { en ré mineur, K. } 466 \text {, } \\
\text { Deuxième mouvement }\end{array}$ & Weinreich Otto & Triphonola \\
\hline$?$ & $\begin{array}{l}\text { Concerto } n^{\circ} 23 \text { en La majeur, K. } 488 \text {, } \\
\text { Deuxième mouvement }\end{array}$ & Reinecke Carl & Triphonola \\
\hline$?$ & Concerto $n^{\circ} 25$ en Do majeur, K. 503 & Singer Otto & Triphonola \\
\hline$?$ & Concerto $n^{\circ} 26$ en Ré majeur, K. 537 & Pathy Ilonka von & Triphonola \\
\hline$?$ & $\begin{array}{l}\text { Concerto } n^{\circ} 26 \text { en Ré majeur, K. } 537 \text {, } \\
\text { Deuxième mouvement }\end{array}$ & Reinecke Carl & Triphonola \\
\hline$?$ & $\begin{array}{l}\text { Concerto } n^{\circ} 26 \text { en Ré majeur, K. } 537 \text {, } \\
\text { Deuxième mouvement }\end{array}$ & Silber Sidney & Recordo \\
\hline$?$ & Concerto $n^{\circ} 27$ en Si bémol majeur, K. 595 & Bachmann Walter & Triphonola \\
\hline$?$ & $\begin{array}{l}\text { Concerto } n^{\circ} 23 \text { en La majeur, K. } 488 \text {, } \\
\text { Troisième mouvement }\end{array}$ & Pugno Raoul & Virtuola \\
\hline
\end{tabular}


style. $\gg^{11}$ Le musicien est en effet considéré comme un représentant caractéristique du piano du début du $\mathrm{XX}^{\mathrm{e}}$ siècle, dans la mesure où son style de jeu présente la plupart du temps une flexibilité rythmique importante, utilise le rubato et la dislocation des accords ${ }^{12}$ traits typiques de la période. ${ }^{13}$ Ces éléments, considérés comme de mauvais goût par Schonberg, ont disparu des pratiques pianistiques au moment où ce dernier s'exprime. De même, dans la littérature musicologique récente consacrée aux styles de piano de la fin du XIX ${ }^{\mathrm{e}}$ siècle et du début du $\mathrm{XX}^{\mathrm{e}}$ siècle, Dohnányi est traité de concert avec les autres grands instrumentistes de la période.$^{14} \mathrm{Et}$ de fait, ces pianistes ne sont généralement pas considérés comme des interprètes adéquats pour Mozart. Or, il me paraît que cette captation témoigne d'une recherche d'un ton de conversation intime entre le pianiste et l'orchestre, que les interprètes mettent en évidence le découpage musical en phrases successives bien délimitées et que le soliste chante avec poésie, mais retenue. Ces aspects sont surprenants parce qu'on ne s'attend pas à les trouver chez un tel pianiste, et ils le sont d'autant plus qu'ils caractérisent le style d'interprétation « mainstream » des concertos pour piano de Mozart durant une bonne partie du XX $\mathrm{X}^{\mathrm{e}}$ siècle, et qui nous est aujourd'hui encore familier.

J'aborderai cette thématique en plusieurs étapes. Je parlerai d'abord brièvement de la réception de Mozart par Dohnányi et de la place qu'a eue l'œuvre du compositeur viennois d'adoption dans la trajectoire de pianiste de Dohnányi. Je m'intéresserai ensuite au style de jeu du musicien hongrois et de son orchestre dans le Concerto en Sol. Pour ce faire, je le mettrai en relation avec celui d'une interprétation de la seconde moitié du XX $\mathrm{XX}^{\mathrm{e}}$ siècle, celle de Murray Perahia. J'effectuerai ensuite une lecture des critiques parues dans la presse des disques Columbia. Dans la continuité, je proposerai un faisceau explicatif pour rendre compte des options interprétatives de Dohnányi. Je comparerai également brièvement la captation du Concerto en Sol avec celles des concertos K. 271 et K. 503. Pour terminer, je traiterai de façon plus générale de la postérité de l'enregistrement Columbia. En effet, il ouvre la voie à une série de disques 78 tours qui comprennent la majorité des concertos pour piano de Mozart, interprétés par d'autres musiciens, et qui ont certainement favorisé l'établissement de ce segment de l'œuvre du compositeur dans le répertoire de concert.

11. Harold C. Schonberg, The Great Pianists from Mozart to the Present (New York : Simon and Schuster, $\left.1966\left[{ }^{1} 1963\right]\right), 344$.

12. Je traduis le terme anglais de «dislocation » utilisé par Neal Peres da Costa, Off the Record. Performing Practices in Romantic Piano Playing (Oxford : Oxford University Press, 2012), 45-46. L'auteur définit la dislocation comme une disjonction temporaire des deux mains. Il articule également ce concept avec ceux de rubato et d'arpégiation.

13. Sur ces aspects, voir entre autre le travail fondateur de Robert Philip, Early Recordings and Musical Style. Changing Tastes in Instrumental Performance, 1900-1950 (Cambridge : Cambridge University Press, 1992), 6-69. Le libellé de «pianiste romantique » n'est bien entendu pas un concept musicologique, dans la mesure où il n'est pas strictement défini, n'a pas d'objet clairement circonscrit et n'a, en tous cas à ce jour, pas rencontré de consensus parmi les chercheurs.

14. Voir par exemple précisément da Costa, Off the Record, 47. 
Dans la littérature musicologique, le style de jeu du pianiste Dohnányi a commencé à être étudié par László Stachó dans un article. ${ }^{15}$ L'auteur traite précisément des traits typiques de la pratique pianistique du début du $\mathrm{XX}^{\mathrm{e}}$ siècle que j'ai mentionnés. Il signale des moments du premier mouvement du Concerto en Sol comme exemples de la tendance à se précipiter vers une note ou un accord accentué ou souligné, et à la ou le prolonger. ${ }^{16}$ Les nombreuses cadences que Dohnányi a écrites pour les concertos de piano de Mozart ont été étudiées par Deborah Kiszely-Papp. ${ }^{17}$

Dans la littérature mozartienne, seules deux contributions traitent substantiellement de l'interprétation de la musique pour piano du compositeur durant la première moitié du $\mathrm{XX}^{\mathrm{e}}$ siècle. Il s'agit des articles « Freedom and Restraint in Recordings of Mozart » de Robert Philip et «"C'est tout Don Juan qui est là" : la Fantasia K. 475 da Leschetizky a Sokolov » de Alfonso Alberti. ${ }^{18}$ Aucun des deux ne parle de Dohnányi ou du Concerto en Sol.

\section{Dohnányi et Mozart}

Dans une conférence sur le romantisme des sonates pour piano de Beethoven prononcée à l'Université de l'Ohio en 1948, Dohnányi affirme : «Classicism is law and order. Classical music is placed on formal elements, is of symmetrical proportions, is impersonal, objective »; de même que : «The greatest Classical composer is Mozart, the most significant Romantic is Schumann, and between these two poles stands Beethoven. ${ }^{19}$ Le pianiste ajoute que Beethoven conjugue le classicisme et le romantisme et qu'il est le plus grand peut-être pour cette raison. Ces vues n'ont rien d'original, elles se rattachent à l'axe le plus marquant de la réception de Mozart depuis le XVIII siècle. ${ }^{20}$ Autre idée commune, qui apparaît lors de la réponse de Dohnányi à une question du journaliste, critique et

15. László Stachó, « Structural Communication and Predictability in the Performance Style of Bartók and Dohnányi », Studia Musicologica 53/1-3 (March 2012), 171-186. Pour les curieux, il faut signaler deux textes de Zoltán Kocsis, « Dohnányi and Bartók as Performers », The Hungarian Quarterly 35/134 (1994), 149-153. ; «Dohnányi plays Dohnányi », The Hungarian Quarterly 45/175 (2004), 132-138.

16. Voir Stachó, « Structural Communication and Predictability », 181.

17. Deborah Kiszely-Papp, «Zenekari és improvizációs elemek Dohnányi zongoramuzsikájában », Dohnányi Évkönyv 2004, éds. Márta Sz. Farkas et László Gombos (Budapest : MTA Zenetudományi Intézet, 2005), 49-57. ; eadem, « Transcending the Piano: Orchestral and Improvisational Elements in Dohnányi's Piano Music », in Perspectives on Ernst von Dohnányi, 102-108.

18. Robert Philip, «Freedom and Restraint in Recordings of Mozart », in Words About Mozart. Essays in Honour of Stanley Sadie, éd. Dorothea Link (Woodbridge : The Boydell Press, 2005), 121-138. ; Alfonso Alberti, " "C'est tout Don Juan qui est là" : la Fantasia K. 475 da Leschetizky a Sokolov », in Interpretare Mozart. Atti del Convegno, éds. Mariateresa Dellabora, Guido Salvetti, Claudio Toscani (Lucca : Libreria Musicale Italiana, 2007) = Strumenti della ricerca musicale, 12, 115-125.

19. Cité par Ilona von Dohnányi, Ernst von Dohnányi, 217.

20. Pour la caractérisation de Mozart en tant que compositeur «classique », son origine et son développement, voir Gernot Gruber, Mozart und die Nachwelt (Munich : Piper, 1987), 19sqq. 
écrivain Dénes Szánthó en 1942 sur ces œuvres non publiées : «In the hands of a musician every line of Mozart is a heavenly toy. $»^{21}$ Toutefois, le musicien, comme beaucoup d'autres alors, considère l'œuvre pour piano de Mozart comme inégale. Il retient surtout les fantaisies. ${ }^{22}$ Du point de vue plus spécifique de l'interprétation, une autre remarque de la fin de sa vie, est intéressante. Selon lui, pour donner plus de vie « [...] Mozart's music can be viewed as a mosaic requiring new color or light on each segment of a theme. $»^{23}$

Dohnányi joue Mozart depuis son enfance. Il tient la partie de piano du Quatuor avec piano $n^{\circ} 1$ en sol mineur, K. 478, lors de sa première apparition publique à l'âge de neuf ans. Par la suite, l'œuvre pianistique du compositeur tient une bonne place dans son répertoire, et même une place plus importante que chez la plupart des virtuoses du début du $\mathrm{XX}^{\mathrm{e}}$ siècle. ${ }^{24}$ Durant cette période, la musique pour piano de Mozart est également défendue par Carl Reinecke et Ferruccio Busoni par exemple. ${ }^{25}$ Dohnányi joue les sonates $n^{\circ} 11$ en La majeur, K. 331 et $n^{\circ} 15$ en Fa majeur, K. 533/494, cette dernière étant plutôt alors rarement interprétée, ainsi que la Sonate pour deux pianos en Ré majeur, K. 448. Il donne également des pièces isolées, telles que le Rondo en la mineur, K. 511. Parmi les concertos, Dohnányi effectue un choix tout à fait étonnant. Mis à part le Concerto pour deux pianos $n^{\circ} 10$ en Mi bémol majeur, K. 365, il n'interprète entre 1906 et 1922 que le Concerto en Sol. Entre 1906 et 1939, le pianiste aura joué quarante-quatre fois cette pièce en concert, dont huit fois durant la seule année 1921, essentiellement lors de sa tournée américaine. La focalisation sur cette œuvre est d'autant plus étonnante qu'elle est a priori dépourvue du caractère «tourmenté », voire démoniaque, qu'ont voulu voir dans certaines pièces de Mozart les hommes du XIX ${ }^{\mathrm{e}}$ et du début du $\mathrm{XX}^{\mathrm{e}}$ siècle, et qu'ils ont par-là même chéries. ${ }^{26}$ Cette période a bien

21. «Dohnányi on Music », in Perspectives on Ernst von Dohnányi, 186. L'idée du « heavenly toy » renvoie en premier lieu à la réception particulière de l'enfant Mozart. Le texte de William Stafford, «The Evolution of Mozartian Biography », in The Cambridge Companion to Mozart, éd. Simon P. Keefe (Cambridge : Cambridge University Press, 2003), 200-212., constitue une bonne synthèse sur la question.

22. Dohnányi formule cette opinion dans une réponse à Harriette Brower concernant la pédagogie du piano au début des années 1920. Voir «Dohnányi as Teacher», in Perspectives on Ernst von Dohnányi, 258.

23. C'est là un conseil de Dohnányi à son élève Catherine A. Smith, « Dohnányi as Teacher », in Perspectives on Ernst von Dohnányi, 249.

24. Voir Ilona Kovács, « Dohnányi Ernő zongoraművészi pályája. I. rész: 1897-1921 », « II. rész: 19211944 », in Dohnányi Évkönyv 2005, éds. Márta Sz. Farkas et László Gombos (Budapest : MTA Zenetudományi Intézet, 2006), 63-150. ; Dohnányi Évkönyv 2006/7 (Budapest : MTA Zenetudományi Intézet, 2007), 303-360.

25. Voir notamment Carl Reinecke, Zur Wiederbelebung der Mozart'schen Clavier-Concerte. Ein Wort der Anregung an die clavierspielende Welt (Leipzig : Gebrüder Reinecke, 1891). Ce dernier a également enregistré plusieurs rouleaux pour piano avec de la musique de Mozart. Voir mon Tableau 1 pour les concertos. Busoni inclut dans son répertoire plusieurs concertos pour piano de Mozart dès 1873. Voir Alexander Hyatt King, Mozart in Retrospect. Studies in Criticism and Bibliography (Londres : Oxford University Press, 1955), 47. Le pianiste italien a réalisé en 1919 un enregistrement discographique pour la Columbia, malheureusement perdu, probablement du second mouvement du Concerto $n^{\circ} 9$ en Mi bémol majeur, K. 271, dans un arrangement personnel.

26. Voir par exemple Gernot Gruber, « Gibt es eine Quintessenz der Wirkungsgeschichte Mozarts ? », in Das Phänomen Mozart im 20. Jahrhundert. Wirkung, Verarbeitung und Vermarktung in Literatur, Bildender Kunst und in den Medien. Gesammelte Vorträge des Salzburger Symposions 1990, éds. Peter Csobádi, Gernot Gruber et alii (Anif/Salzbourg : Verlag Ursula Müller-Speiser, 1991), 24. 
entendu préféré le Concerto pour piano $n^{\circ} 20$ en ré mineur, K. 466. Dès 1923 , Dohnányi ajoute à son répertoire, mais de façon sporadique, les concertos $n^{\circ} 9$ en Mi bémol majeur, K. 271, $n^{\circ} 20$ en ré mineur, K. 466, $n^{\circ} 23$ en La majeur, K. 488, $n^{\circ} 25$ en Do majeur, K. 503 et $n^{\circ} 26$ en Ré majeur, K. 537. En 1941 et 1942, il joue une intégrale des concertos pour la Radio Hongroise, dont nous n'avons malheureusement conservé aucune trace sonore. ${ }^{27} \mathrm{Ce}$ cycle suit de peu celui joué par Nadia Reisenberg pour la station de radio new-yorkaise WOR en 1939 et 1940. Des pièces de musique de chambre avec piano figurent également régulièrement au répertoire de Dohnányi. Ajoutons qu'en 1921 Bartók a entendu Dohnányi jouer le Concerto en Sol et qu'il en a été ravi. ${ }^{28}$

Un autre pan important du travail effectué par Dohnányi autour de l'œuvre de Mozart concerne, je l'ai dit, les cadences qu'il a écrites pour les concertos de piano. Il faut encore signaler que le musicien a publié une édition instructive de la Fantaisie en ré mineur, K. 397 en 1919 et que, comme Mozart, il a composé une série de variation sur le thème de $A h$ ! vous dirais-je maman, op. $25 .{ }^{29}$

\section{L'enregistrement Columbia du Concerto en Sol}

La méthode que je vais utiliser pour analyser les aspects que je considère comme étonnants dans l'enregistrement de Dohnányi correspond en quelque sorte à la façon dont je l'ai abordé en tant qu'auditeur et chercheur. Né à peu près au milieu de la seconde moitié du $\mathrm{XX}^{\mathrm{e}}$ siècle, j'ai fait connaissance avec les concertos pour piano de Mozart via les partitions, le concert et les enregistrements couramment diffusés par l'industrie du disque à la fin du siècle. ${ }^{30}$ Parmi les interprétations sur « instruments modernes » que je connais, j'ai fréquenté par goût, plus que d'autres, celles de Murray Perahia, qui en a réalisé une intégrale accompagné par l'English Chamber Orchestra qu'il dirige lui-même. ${ }^{31}$ L'enregistrement du Concerto en Sol date de 1980. Nonobstant, cette intégrale constitue un bon point de comparaison, dans la mesure où elle est encore une des plus admirées aujourd'hui et a largement été diffusée. C'est avec ces représentations sonores que j'ai pris connaissance de l'enregistrement de Dohnányi, et les similitudes m'ont plus interpellé que les dif-

27. Voir Kiszely-Papp, « Transcending the Piano », 103.

28. Voir « Béla Bartók on Dohnányi », in Perspectives on Ernst von Dohnányi, 79.

29. W056 et W125 respectivement dans la liste de Grymes, Dohnányi. A Bio-Bibliography.

30. Sans compter évidemment tous les aspects proprement culturels autour de ces œuvres, qui ont dû avoir un impact sur moi.

31. Cette intégrale a été enregistrée entre 1975 et 1988 et est parue, pour la plus grande partie de ses albums, d'abord en LPs chez CBS. Elle a été republiée en CDs dans de multiples éditions chez Sony. C'est via une d'entre elles que j'ai écouté et analysé le Concerto en Sol (Murray Perahia plays Mozart. The Piano Concertos, 12 CDs, Sony Classical, CDs 886919141122, 2012). 
férences. En outre, j'ai écouté cette captation avant de lire les critiques qui en rendent compte. ${ }^{32}$

Examinons les uns après les autres les paramètres que je cherche à mettre en évidence. Je procéderai via une analyse d'extraits représentatifs, extraits à cause des dimensions forcément restreinte de ce texte.

Commençons par le second groupe thématique du soliste, rejoint ensuite par l'orchestre, dans l'exposition du premier mouvement du concerto. La partition de cet extrait est reproduite dans l'Exemple 1. Dans l'enregistrement de Dohnányi, il débute à 3'11', dans celui de Perahia, à 3'09'. Les deux pianistes produisent un touché délicat dans l'énoncé du thème à la main droite et leur accompagnement est discret. Dans les gammes ascendantes, les staccatos indiqués par Mozart à la mes. 116 sont réalisés de façon légère et en diminuendo sur la fin, le jeu appuis-contre-appuis rythmiques est subtil. Lors de leurs entrées à la mes. 118, les cordes sont souples dans leur accompagnement, et lorsque les vents reprennent la tournure cadentielle (mes. 121 sqq.) que le piano avait exposé au sein de son thème (mes. 114-115), les pianistes s'insèrent dans le discours en «titillant» leur partenaire. Nous avons ici affaire au ton qu'on appelle à défaut de mieux de « conversation intime » entre les instrumentistes. Il faut également relever le changement de couleur opéré par les interprètes au moment du passage au mode mineur à la mes. 126. Toutefois, dans le thème proprement dit, Dohnányi et Perahia choisissent des micros-phrasés différents. Ceux du pianiste hongrois correspondent mieux aux indications de Mozart.

Dès la mes. 124 l'orchestre qui accompagne Dohnányi accélère. Il s'agit là également d'une tendance courante de l'interprétation des ensembles instrumentaux d'avant la Seconde Guerre Mondiale et qui a peu à peu disparue. ${ }^{33}$ L'orchestre se produit en version de chambre. $\mathrm{Si}$, à l'écoute, cette composante n'est pas forcément évidente, nous en avons une quasi confirmation grâce à la critique du concert du 15 juin parue dans le Morning Post le lendemain, qui, précisément, insiste sur cet aspect (voir ci-dessous). L'orchestre qui accompagne Dohnányi est donc du même type que celui de Perahia. De fait, un ensemble instrumental de chambre semble être un meilleur partenaire pour dialoguer ainsi qu'un orchestre symphonique. De façon générale, l'orchestre de Dohnányi est loin d'être techniquement parfait, mais il nous faut prendre garde de ne pas considérer les accélérations, les traits

32. Par ailleurs, il me paraît que nous apprécions et jugeons les interprétations forcément via une écoute comparative. La partition, si elle est sollicitée, permet d'instaurer un rapport critique avec les interprétations. La relation au texte musical ne me paraît pas première pour l'appréhension de ce que nous considérons comme l'œuvre, dans la mesure où ce texte n'est qu'un codage incomplet : il ne contient que les informations non redondantes dans un langage musical donné et culturellement informé. De plus, l'appréciation des interprétations fait intervenir une dialectique norme-écart, avec ici comme norme celles auxquelles nous sommes accoutumées.

33. Voir Philip, Early Recordings, 6-69. 
EXemple 1 Mozart, Concerto pour piano $n^{\circ} 17$ en Sol majeur, K. 453, Allegro, mes. 110-126
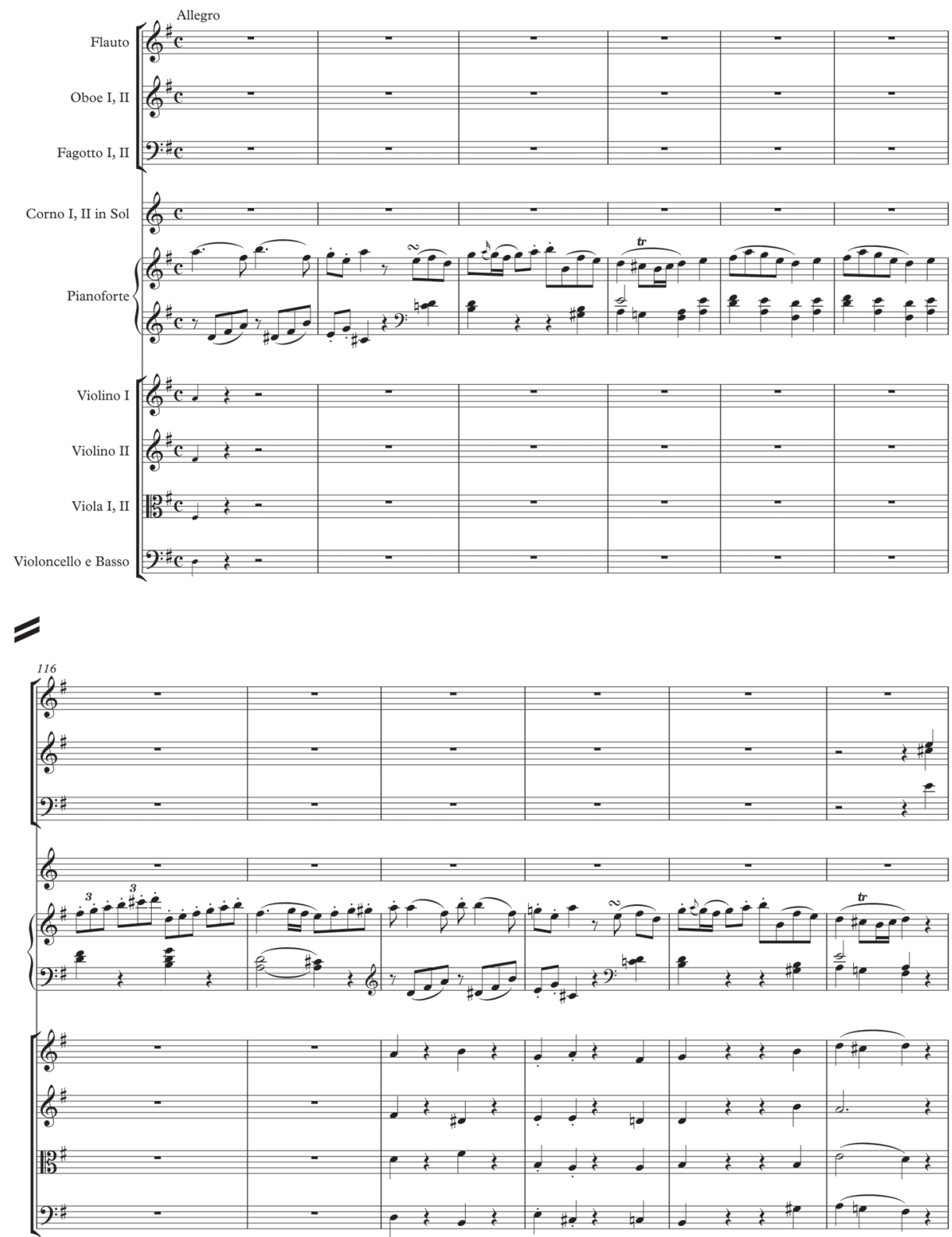
EXEMPLE 1 (Continuation)

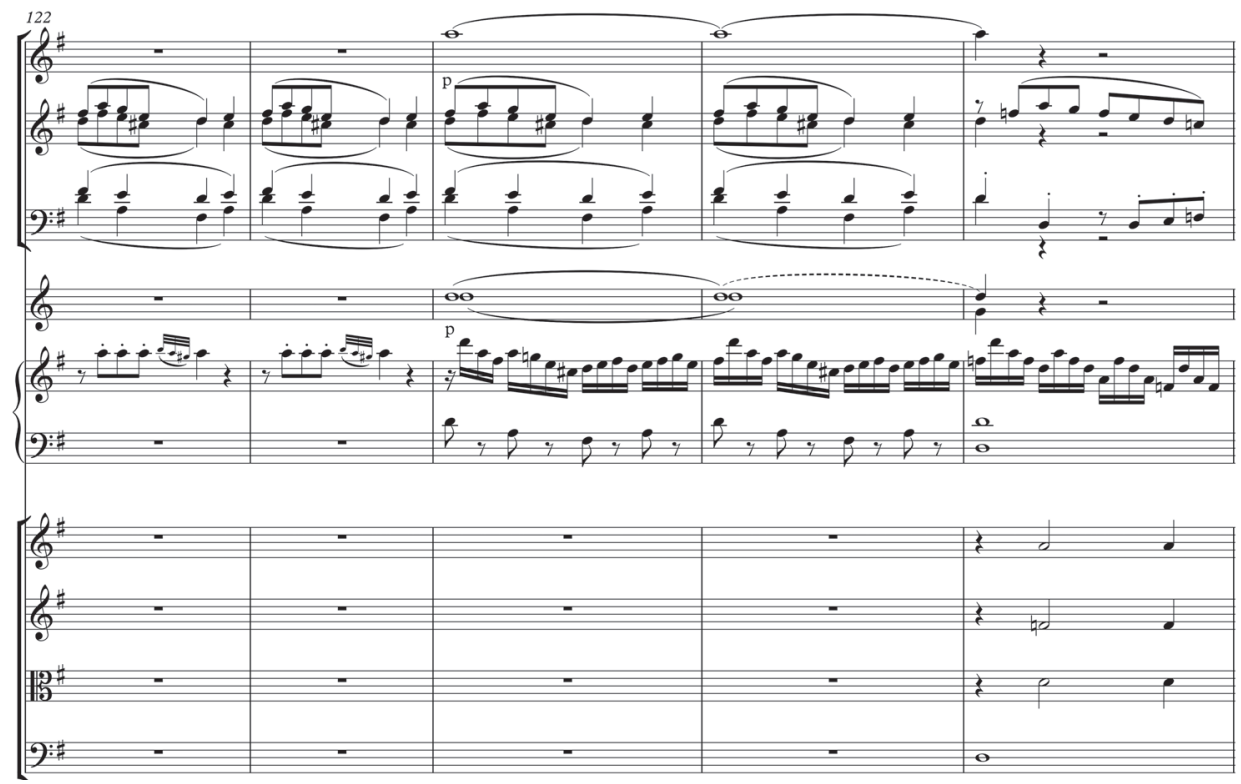

de gammes qui fusent et quelques fins de parties bien appuyées comme des déficiences. Ce sont là des éléments assumés et typique de la période.

Les interprétations du début du troisième mouvement, à savoir le thème qui sera ensuite varié, constituent un bon exemple de mise en évidence des phrasés à des fins de clarification formelle. On trouvera le texte musical de ce début dans l'Exemple 2. Dans les deux enregistrements, la structure antécédent - conséquent des quatre fois huit mesures qui constituent ce thème est soulignée. Dohnányi et son ensemble instrumental le réalisent essentiellement grâce à la dynamique dans les seize premières mesures orchestrales et via à un jeu combinant dynamique et agogique dans les seize suivantes. Perahia et son orchestre mettent en évidence cette structure grâce à la dynamique tout au long des trente-deux mesures et ils cèdent légèrement toutes les huit mesures. Le tempo de référence choisi par Dohnányi est légèrement plus rapide.

Dans les variations, le style de jeu de Dohnányi et de son orchestre est infléchi. La souplesse du tempo est plus marquée, quelques phrasés semblent " heurtés » et certains traits de virtuosité paraissent à notre oreille « moins soignés ». Il faut peut-être précisément chercher dans le caractère virtuose des variations la cause de ces différences.

Les exécutions du mouvement lent présentent également des similitudes. Le premier solo du piano et les quelques mesures qui suivent en sont une bonne illustration. Ce passage figure dans l'Exemple 3. Il apparaît à 2'06' dans l'enregis- 
EXemple 2 Mozart, Concerto pour piano $n^{\circ} 17$ en Sol majeur, K. 453, Allegretto, mes. 1-32
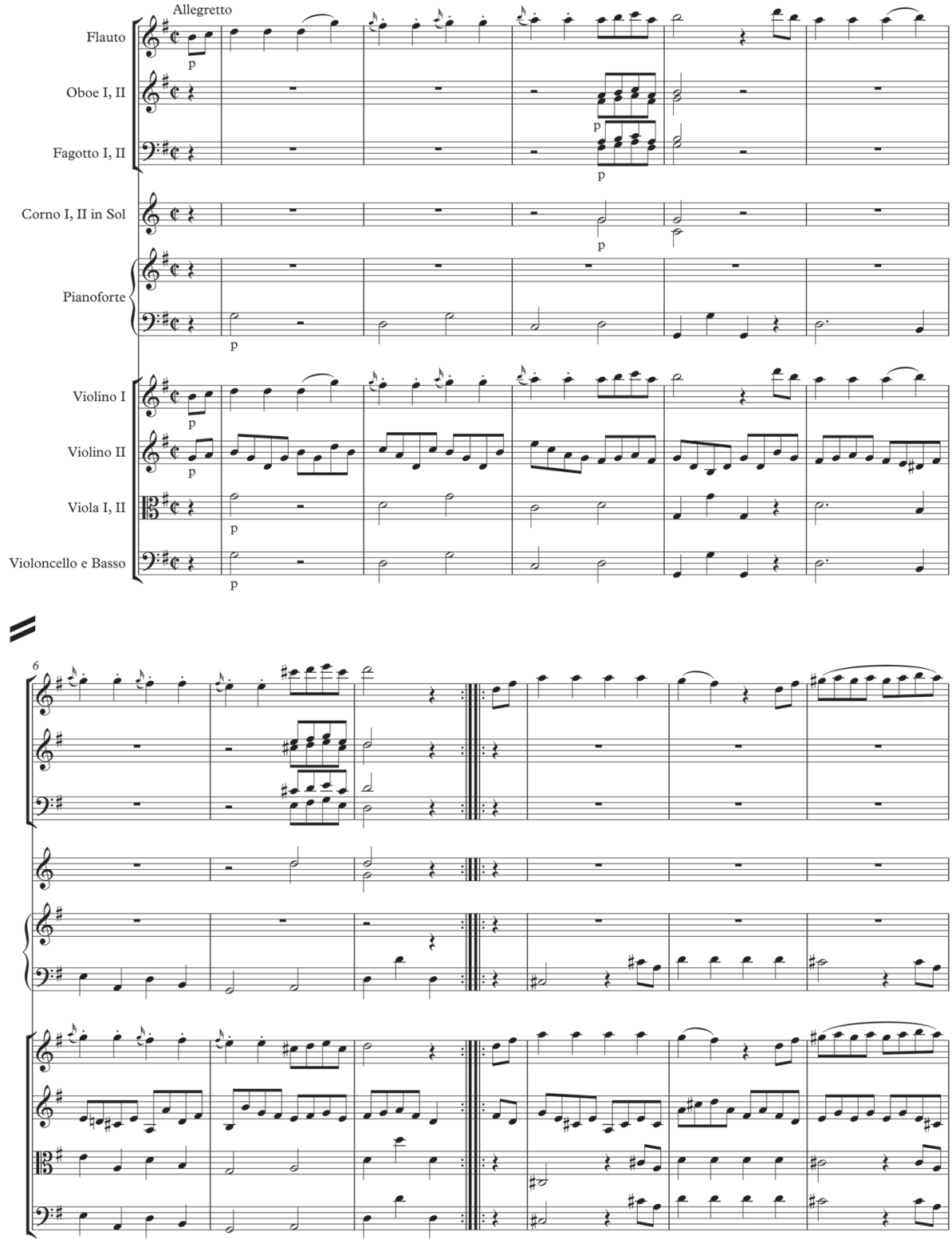
Vers le style d'exécutuion «mainstream» des concertos pour piano de Mozart 209

EXEMPLE 2 (Continuation)
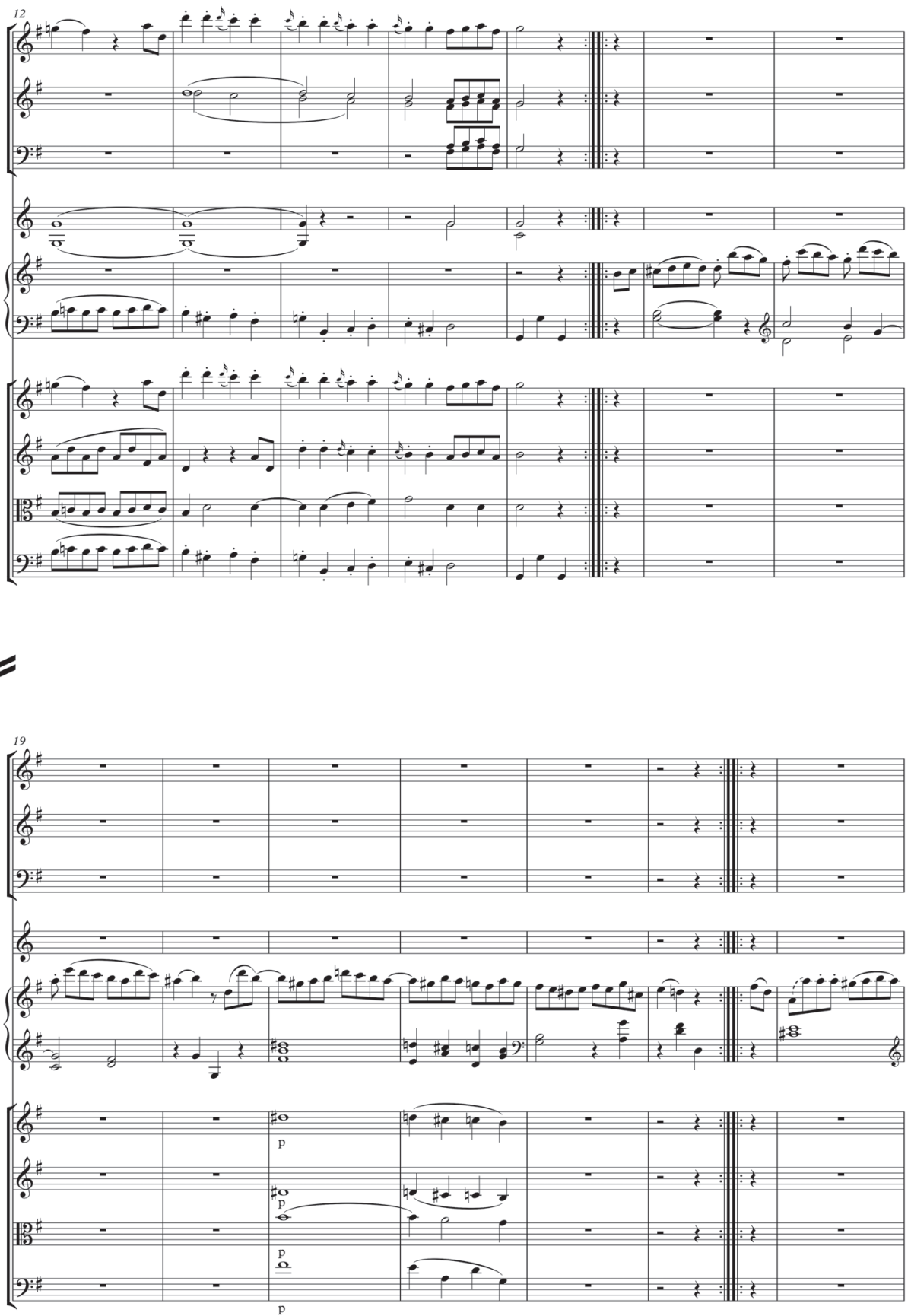
EXEMPLE 2 (Continuation)

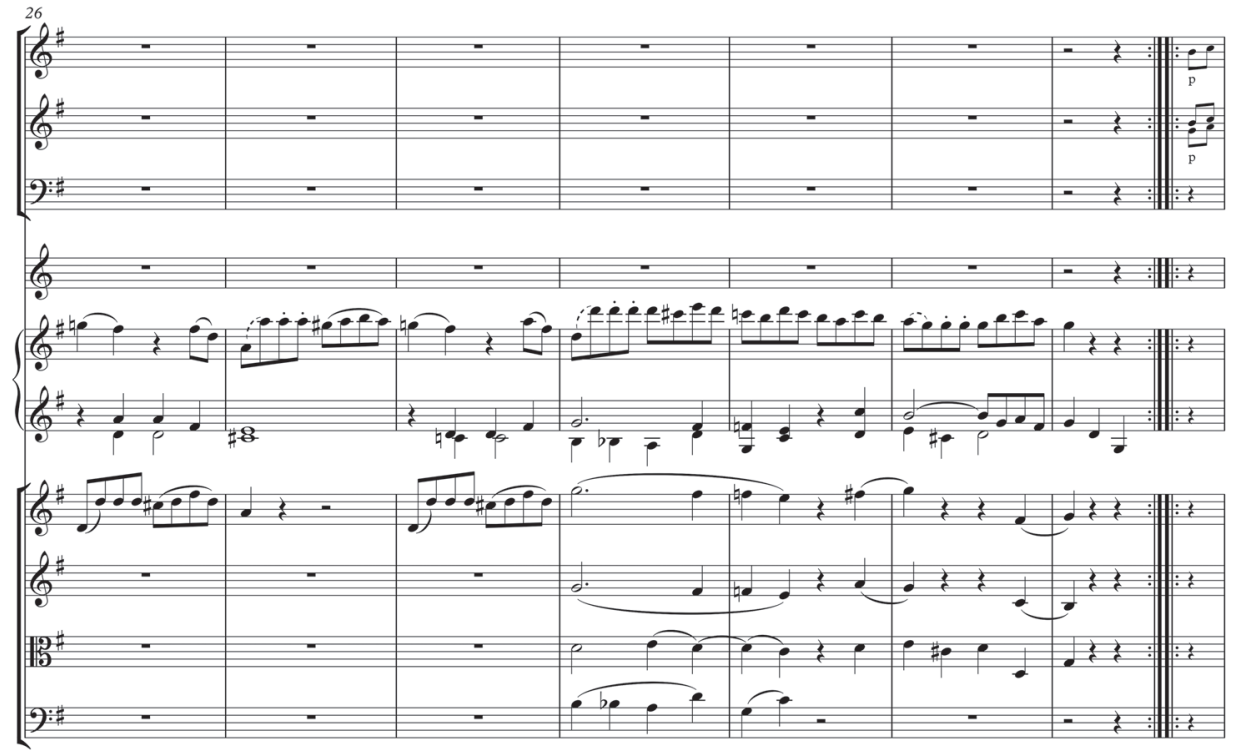

trement de Dohnányi et à 1'55' dans celui de Perahia. Dans le solo, les pianistes font chanter leur instrument, avec un équilibre entre poésie et retenue : la main gauche reste effacée, tandis que la main droite, si elle met en évidence la mélodie, se maintient également dans une nuance piano voire pianissimo. Le touché est par ailleurs très soigné. Le $m i$ aigu de la mes. 33, climax mélodique, n'est pas joué d'une manière qui le mettrait trop en évidence. Enfin, les interprètes achèvent leur phrase par un pianissimo malgré les grands accords de la main gauche. La suite, qui module directement en sol mineur et introduit l'orchestre, est jouée comme un drame intérieur, mais qui reste pudique. La parenté me paraît frappante malgré le rubato et une attitude moins littérale dans l'exécution des valeurs rythmiques dans le solo chez Dohnányi.

L'examen de ces extraits a en outre montré que Dohnányi et son orchestre sont tout aussi respectueux du texte musical proprement dit de Mozart que le sont Perahia et l'English Chamber Orchestra. Le pianiste hongrois n'ajoute notamment aucun ornement de son cru. C'est là également un aspect qui «ne va pas de soi » au vu de la perception que nous pouvons avoir des pianistes du début du $\mathrm{XX}^{\mathrm{e}}$ siècle. ${ }^{34}$

34. Cependant, les partitions des concertos de Mozart sur lesquelles travaillait le pianiste, conservées dans la Collection Dohnányi, attestent de réécritures de passages, de modification de notes et d'ajouts d'ornements. Voir James A. Grymes, «The Ernst Dohnányi Collection at the Florida State University », Notes 55/2 (December 1998), 335-337. 
EXemple 3 Mozart, Concerto pour piano $n^{\circ} 17$ en Sol majeur, K. 453, Andante, mes. 30-39
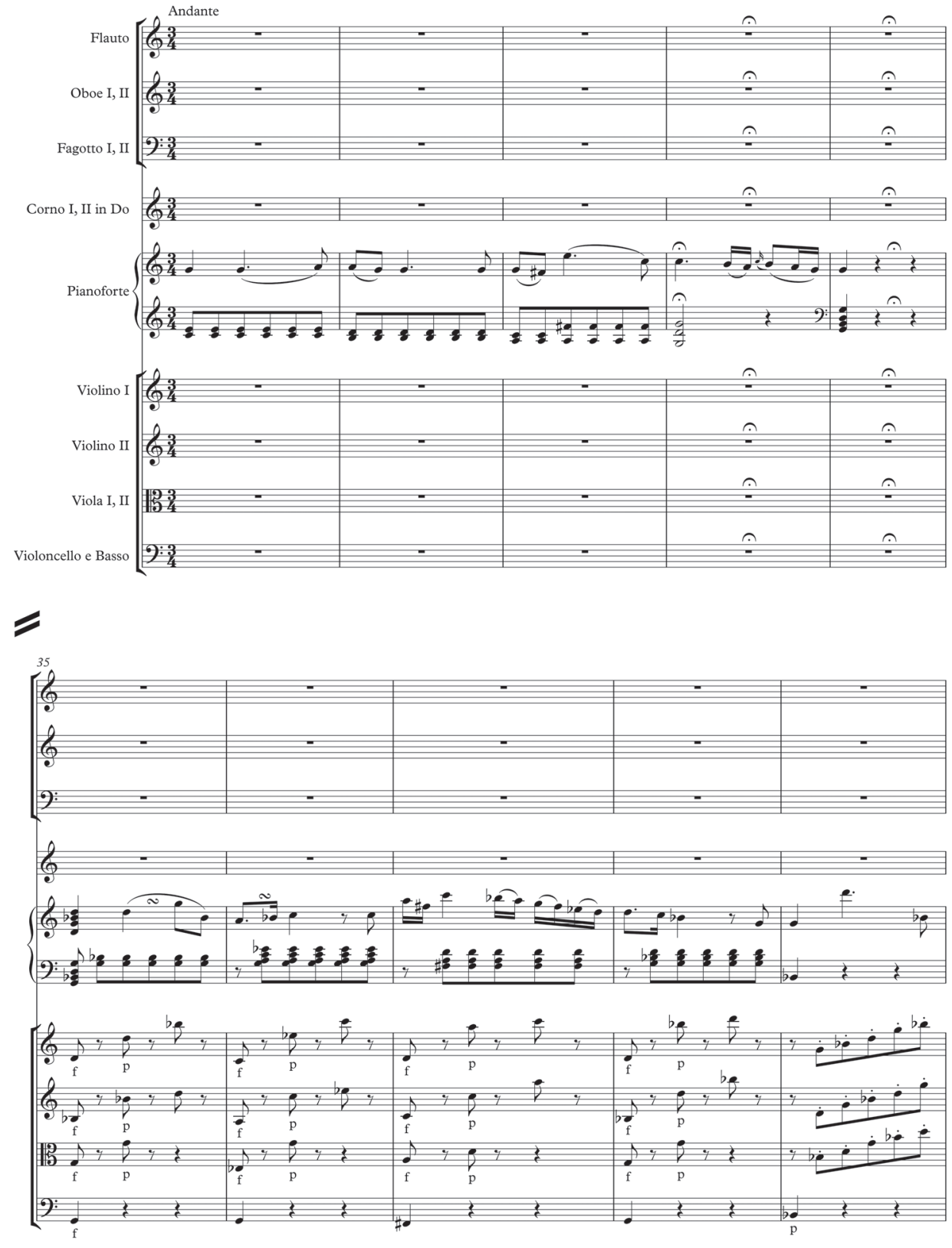
Je l'ai dit, Dohnányi et son orchestre jouent dans un tempo souple. Bien enten$\mathrm{du}$, Perahia et son orchestre privilégient une battue plus stable. Dohnányi recourt au rubato et à la dislocation rythmique, tandis que ces éléments ont disparu chez Perahia. Les cadences de solistes témoignent également d'une approche différenciée. Si le pianiste américain joue les cadences laissées par Mozart, les fins du premier et du deuxième mouvement en nécessitent, le musicien hongrois interprète les siennes. Dohnányi avait publié des cadences pour ce concerto dès 1909. Dans l'enregistrement de 1928, il prend des libertés par rapport à cette version. ${ }^{35}$ La question des cadences ayant déjà été traitée, je ne m’y attarde pas plus ici.

\section{Réception critique de l'enregistrement}

Cette réalisation de la Columbia a fait l'objet de quelques comptes-rendus critiques. Le concert londonien de juin donné en parallèle à la session d'enregistrement également. Commençons par ceux-ci. Dans les deux critiques recensées, le débat se focalise sur la direction de l'orchestre prise en charge par le pianiste lui-même. C'est peut-être un signe que la pratique n'est pas encore pleinement entrée dans les mœurs. Ainsi, on peut lire dans l'édition du 16 juin 1928 de The Morning Post :

The Mozart Concerto, in which Dohnanyi [sic !] was soloist, was in my opinion an unsatisfactory performance, chiefly because it was conducted from the piano, and because the leader could not possibly see the pianist's occasional instructions. At the beginning of the first movement there was considerable confusion. ${ }^{36}$

Et dans The Times du même jour :

Mr. Dohnanyi [sic !] is very well known and has been long appreciated in this country both as pianist, and composer. In this programme, which began ceremoniously with the National Anthems of England and Hungary, followed appropriately by Liszt's symphonic poem, "Les Préludes," his art was displayed in both these capacities as well as in that of conductor. He gave a deliciously delicate interpretation of Mozart's pianoforte concerto in $\mathrm{G}$ accompanied by a small number of the orchestra, whom he directed from his seat at the piano.

35. Voir Deborah Kiszely-Papp, « Transcending the Piano », 105-108. ; eadem, «Zenekari és improvizációs elemek Dohnányi zongoramuzsikájában », Dohnányi Évkönyv 2004, 50-54. Je remercie Eva Krasznai Starobinski et Georges Starobinski, qui m'ont aidé à prendre connaissance de la version hongroise du texte de Kiszely-Papp.

36. Cité par Ilona Kovács, « Dohnányi Ernő zongoraművészi pályája. II. rész », 310-311. 
They accompanied perfectly, but there were one or two moments in the Tutti passages where the plan was not entirely satisfactory. The wind players were perhaps too far from the first violins to be able to do without a visible beat. ${ }^{37}$

Si l'appréciation critique sur la réussite du concert est opposée dans les deux articles, ils se rejoignent sur quelques imperfections de mise en place. Peu d'informations sont toutefois à tirer sur l'interprétation elle-même du concerto.

Une première critique discographique, non signée, paraît en février 1929 dans The Gramophone :

The concerto is in an album, with a pamphlet of notes. The first and second movements take three sides each, and the last movements two. Dohnanyi [sic !] conducts from the piano, as I think he has done at a London concert. When you and the orchestra know each other's every lift of the eyelid, that can be done safely; but I once saw a certain lady pianist-conductor (she really is a pianist) try to lead the orchestra from the piano, and succeed only in leading them astray. Some of her movements, made probably involuntarily, were apparently interpreted by the orchestra as nods and becks for them; the result was sad. In older days - up to the early nineteenth century - the conductor commonly sat at the piano, beating time now and again, and playing a little in case of need, to reassure, support or animate the players. Dohnanyi makes a good job of it, and of his playing, though his brilliance is a trifle hard. In the slow movement he does not quite give us the gently reflective tone of the music. Where gaiety and force are in order, he is a very live wire. The dapper bounce of the last movement (variations) finds soloist and orchestra in their best vein, though even here there is a slight feeling of "forcing the pace" in places. The pianist is just a little over-eager, I feel; but the spirit and address of the music are well conveyed. This is a good record to get if one does not wish at first, at any rate, to buy the whole work. The recording is of a high quality, and in places quite the best. ${ }^{38}$

Ce texte est intéressant à plusieurs égards. Le critique insiste sur l'ampleur de la réalisation discographique en indiquant que l'œuvre occupe quatre disques. En effet, de telles productions sont encore rares. Et il est piquant pour nous, qui disposons d'une quantité considérable d'enregistrements à portée de clic, de lire le conseil de privilégier le dernier disque, celui qui comporte le troisième mouvement de l'œuvre, en cas d'achat partiel. Comme dans les comptes-rendus du concert, le critique traite de la question du pianiste-chef, ici en narrant une mauvaise expérience, je n'y reviens pas. En ce qui concerne l'interprétation proprement dite, le

37. Ibid., 311.

38. The Gramophone 6/69 (February 1929), 398. 
critique juge favorablement pianiste et orchestre dans le troisième mouvement, celui qui est le plus gai. La remarque négative sur le soliste surtout qui force l'allure est révélatrice. En effet, le degré de souplesse dans la gestion du tempo fait débat dans ces années. ${ }^{39}$ Par ailleurs, cette observation corrobore mon impression à l'écoute d'un style d'interprétation légèrement distinct dans ce mouvement. Egalement négative, une facette de l'appréciation générale sur le jeu de Dohnányi, que le critique estime légèrement dur à son goût. Enfin, l'auteur regrette son manque de délicatesse dans le mouvement lent. Ce qualificatif apparaît également dans une autre critique.

Les disques Columbia sortent également en France et deux comptes-rendus, plus brefs, en parlent. Sous la plume de Ch. Reynault, on lit dans le numéro de juillet 1929 de la Revue des machines parlantes :

Indiquons aussi par cette même marque [Columbia] l'enregistrement superbe du Concerto en sol n ${ }^{\circ} 17$, de Mozart, où le chef de l'Orchestre Philharmonique de Budapest, M. Ernst Dohnányi conduit l'exécution tout en tenant lui-même la partie de piano. L'interprétation de cette œuvre est transcendante, étonna[n]te de souplesse, de légèreté et de délicatesse.

La réalisation phonographique est fidèlement rendue par le disque et fait très heureusement ressortir toute la valeur d'un style infiniment séduisant dans ses plus infimes détails. (Col[umbia].) $)^{40}$

Ici, le jugement de valeur est globalement positif. Toutefois, l'élément le plus intéressant est peut-être, au-delà des divergences d'appréciation sur la réussite de l'interprétation dans les critiques citées, la norme esthétique implicite par rapport à laquelle cette lecture est évaluée : les concertos pour piano de Mozart exigent du soliste légèreté et délicatesse. De plus, les éléments peu appréciés par le critique du Gramophone sont précisément ceux qui disparaîtront petit à petit dans le courant du siècle. Faut-il y voir une influence de la pression normative de la critique ?

Le second texte français est dû à Emile Vuillermoz, un des critiques les plus influents de l'époque. Il est paru un mois avant celui de Reynault dans L'Edition musicale vivante. S'il apporte moins d'informations pour notre analyse, il mérite d'être mentionné pour sa thématisation, encore une, de la question du pianiste qui joue tout en dirigeant :

Enfin, les amateurs de concertos pour piano et orchestre feront bien de stocker dans leur collection le Concerto en si [sic !] $n^{\circ} 17$ de Mozart (C[olumbia]) exécuté par Ernst von Dohnanyi [sic !] qui conduit de son clavier l'orchestre phil-

39. Voir Philip, Early Recordings, 7-16.

40. Revue des machines parlantes $\mathrm{n}^{\circ} 116$ (juillet 1929), 312. 
harmonique de Budapest. Ce pianiste chef d'orchestre s'acquitte fort bien de sa double mission. A chaque instant, on trouve dans son exécution des détails pianistiques extrêmement réussis. Cette série est une pièce de qualité qui supporte fort bien l'amplification électrique. ${ }^{41}$

De fait, une norme esthétique qui va dans le sens décrit dans ces critiques et qui corrobore mon écoute semble se dégager des textes musicologiques et musicographiques contemporains. ${ }^{42}$ Dans sa biographie de Mozart, parue une première fois en 1914, Henri de Curzon dit du Concerto n6 en Si bémol majeur, K. 238, qu'il « [...] ne brille pas par la virtuosité, mais par la pureté et la poésie mélodique, par une élégance discrète désormais inséparable du génie de Mozart. ${ }^{43}$ Dans les termes de Hans Engel, l'auteur en 1927 d'une histoire du concerto pour piano allemand de Mozart à Liszt, les derniers mouvements de Mozart sont des « [...] Spätlinge des Rokoko voll Grazie und Charme. »" Dans la littérature anglophone, Eric Blom soutient des propos similaires, dans sa biographie sur Mozart publiée en 1935. Voici ce qu'il affirme précisément à propos du Concerto en Sol, qu'il considère comme une « delightful work » : « This is music of such happiness as Mozart, who is never quite untroubled by conflicting emotions, could produce gently cheerful in the first movement, filled with a quiet gratitude, not without twinges of reminiscent plain, in the Andante, which opens rather like Schubert's song "Du bist die Ruh", and full of a cocksure chirpiness in the finale, which might be a set of variations on a song for Papageno in The Magic Flute. ${ }^{45}$

Comme Henri de Curzon, Arnold Schering relève, en 1905 dans la première étude consacrée au concerto instrumental, la primauté de la mélodie chez Mozart. Ainsi, dans le Concerto n $^{\circ} 20$ en ré mineur, K. 466, « [...] thront über allem der Zauber berückender Kantabilität. $»^{46}$

41. L'Edition musicale vivante $\mathrm{n}^{\circ} 17$ (juin 1929), 23.

42. Je laisse de côté ici, pour une question de place, les réflexions de Hermann Abert sur les concertos pour piano de Mozart. Elles témoignent d'une pensée complexe et nécessiteraient une lecture serrée. Son développement sur la question figure essentiellement dans le Chapitre 39 de sa monumentale biographie sur Mozart. Voir Hermann Abert, W. A. Mozart, éd. Cliff Eisen, trad. Stewart Spencer (New Haven, Connecticut-Londres : 2007 [en allemand : ${ }^{1} 1919$ ]), 870-903. Sur les relations, encore peu étudiées, entre réception et interprétation musicale, voir José A. Bowen, « Finding the Music in Musicology: Performance History and Musical Works », in Rethinking Music, éds. Nicholas Cook, Mark Everist (Oxford : Oxford University Press, 1999), 424-451. ; Daniel Leech-Wilkinson, « Musicology and Performance », in Music's Intellectual History, éds. Zdravko Blažeković, Barbara Dobbs Mackenzie (New York : Répertoire International de Littérature Musicale, 2009), 791-803.

43. Henri de Curzon, Mozart (Paris : Félix Alcan, 1914), 107.

44. Hans Engel, Die Entwicklung des deutschen Klavierkonzertes von Mozart bis Liszt. Mit einem Notenanhang (Leipzig : Breitkopf und Härtel, 1927), 66. (Reprint : Hildesheim-New York : Georg. Olms ; Wiesbaden : Breitkopf und Härtel, 1970.)

45. Eric Blom, Mozart (Londres-New York : J. M. Dent and Sons, E. P. Dutton and Co., 1935), 223.

46. Arnold Schering, Geschichte des Instrumentalkonzerts bis auf die Gegenwart (Leipzig : Breitkopf und Härtel, 1905), 162. 
De plus, l'idée de conversation intime apparaît chez le même auteur, lorsqu'il opère une distinction entre ce concerto et les autres : « Das grosse Konzert in der Don Juan-Tonart D-Moll, dass Beethoven selbst mit Kadenzen versah, ist bis zum heutigen Tage das gefeiertste geblieben, weil es den leichten Konversationston gänzlich meidet und durch harmonische Paarung von Ernst und Milde ersetzt. $»^{47}$

Sur la question de la clarté, Hans Engel dit que Mozart « [...] war überhaupt gegen zu schnellen Tempi und verlangt Deutlichkeit und Nettigkeit [...]. ${ }^{48}$ Il n'est toutefois pas possible de savoir si dans le cas présent l'auteur pense à la clarté formelle ou une netteté d'émission des sons.

S'il semble y avoir concordance entre réception et interprétation musicale, il est impossible de dire si ce lien préexiste à la captation Columbia, étant donné qu'elle est la première. De même, on ne peut pas savoir dans quelle mesure Dohnányi lui-même a contribué à l'émergence de ce style de jeu. Les rouleaux mentionnés qui préservent des interprétations de concertos sont, malgré leur intérêt, peu informatifs sur ces aspects. ${ }^{49}$

Il faut encore tenir compte d'un autre paramètre pour poursuivre la réflexion, celui de la technique de l'enregistrement lui-même et de l'écoute discographique. En effet, dans l'interview de 1942 que j'ai mentionnée, Dohnányi répond également à une question sur l'aspect mécanique que prend l'interprétation musicale. Voici ce qu'il dit à propos de l'enregistrement :

One has to simplify. In order not to become boring one has to make the music artificially uninteresting. To make this paradox clear: the more rubato, the more free and spontaneous fantasizing there is on a gramophone recording, the faster the owner of the recording will develop an aversion to that piece as he puts it on the turntable over and over again. One has to be cool and objective here and this will preserve the performer on the recording from becoming prematurely boring. ${ }^{50}$

Serait-ce là une des causes de ce style de jeu plus mesuré, qui ne correspond pas à l'image habituelle du pianiste Dohnányi ? Pour tenter d'y voir plus clair, un examen comparatif s'impose, celui de l'interprétation du Concerto en Sol avec celles des deux concertos de Mozart joués en public par le pianiste dans sa période américaine dont on possède des captations. 


\section{Les captations tardives de concertos de Mozart}

La mise en relation se révèle globalement peu informative, dans la mesure où le style de jeu du pianiste me paraît globalement similaire. ${ }^{51}$ En particulier, les thèmes dans les premiers mouvements sont joués de la même manière lors des concerts américains que sur les disques Columbia. Dans les deuxièmes mouvements, le rubato et la dislocation des accords sont présents dans des proportions équivalentes. Un seul moment tranche avec le reste : le thème de soliste et ses reprises dans le troisième mouvement du Concerto $\mathrm{n}^{\circ} 9$ en Mi bémol majeur, K. 271. Ici, Dohnányi joue avec une souplesse de tempo considérable et de «brusques » à-coups.

Une analyse des fluctuations de tempo avec le programme Sonic Visualiser a confirmé cette impression auditive. Pour ce faire, j’ai choisi la seconde exposition du thème (mes. 150-184, reproduites dans l'Exemple 4). ${ }^{52}$ La première occurrence (mes. 1-35) s'est révélée inadaptée, dès lors que la première mesure manque dans la captation. La troisième présentation (mes. 304-312 et 365-374) est moins propice à ce type d'examen, parce qu'elle est incomplète et entrecoupée par l'orchestre. Une analyse analogue de l'exposition proprement solistique (mes. 17-32, pour rappel reproduites dans l'Exemple 2) du thème du troisième mouvement du Concerto en Sol permet de mettre en regard les traitements du tempo. ${ }^{53}$ On peut discuter de la pertinence de ce choix pour la mise en relation. En effet, ces deux mouvements ne présentent pas le même caractère, le début du mouvement du Concerto en Sol est noté Allegretto, tandis que la partie retenue du Concerto en Mi bémol doit être jouée Presto ; le Finale (dès mes. 171) du Concerto en Sol est aussi un Presto, mais ici aucun énoncé thématique n'est véritablement pris en charge par le pianiste seul. Aucun autre extrait n'est cependant mieux adapté.

Les résultats de ces analyses sont reportés dans les Tableaux 2 et $3 .^{54}$ Les fluctuations de tempo sont effectivement beaucoup plus importantes dans le Concerto en Mi bémol que dans celui en Sol : les fourchettes de tempos fluctuent entre

51. J'ai écouté et analysé ces captations, conservées sous forme de cassettes DAT, via des fichiers audio WMA que m'a aimablement transmis Veronika Kusz, de l'Institut de musicologie, Centre de recherche des sciences humaines de l'Académie hongroise des sciences à Budapest.

52. Dans le fichier audio, l'extrait débute à 24'38'.

53. Certes, l'orchestre effectue ici quelques interventions en accompagnement. Mais puisque Dohnányi le dirige lui-même (ou puisque ses membres sont certainement tout à fait capables de suivre ici le pianiste par eux-mêmes...) et parce que les partenaires se connaissent bien, on peut supposer que le soliste n'est pas freiné dans ses velléités interprétatives en matière de variation de tempo.

54. J'ai utilisé la version 3.0.3 de Sonic Visualiser. Le «tapping » a été réalisé à $80 \%$ du tempo pour le troisième mouvement de K. 453 et à $50 \%$ pour celui de K. 271. J'ai effectué quelques corrections à différents pourcentages moins élevés. Les graphique ont été générés avec l'option « Tempo (bpm) based on duration since the previous item », ce qui explique les marques de tempo à 0 bpm au début des tableaux. J'ai eu quelques difficultés à placer certaines marques temporelles dans K. 271, à cause de la mauvaise qualité sonore de la captation. En effet, les attaques du piano sont parfois brouillées par l'acoustique généreuse de la salle et la prise de son lointaine. Je remercie Olivier Silberstein pour son aide dans la mise en page des graphiques. 
EXEMPLe 4 Mozart, Concerto pour piano $n^{\circ} 9$ en Mi bémol majeur,

K. 271 , Presto, mes. $150-184$

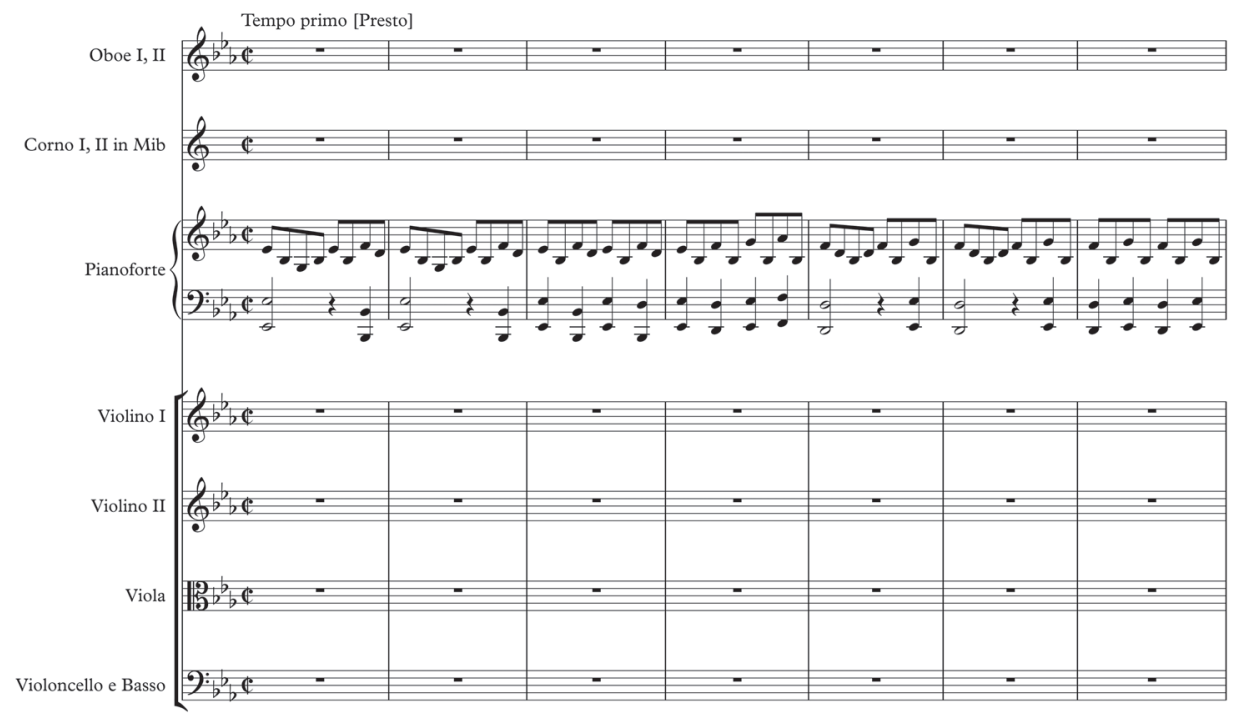

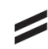

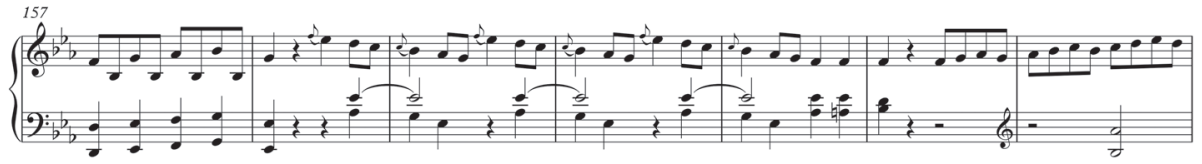

2

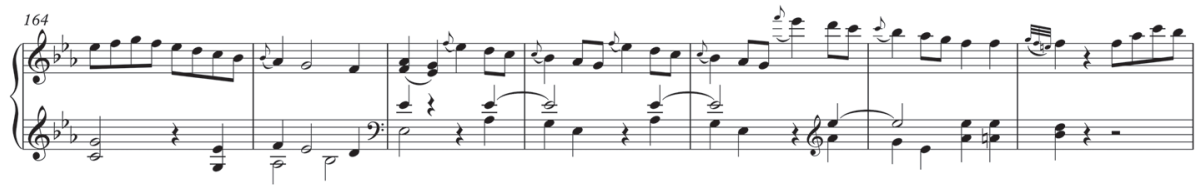

2

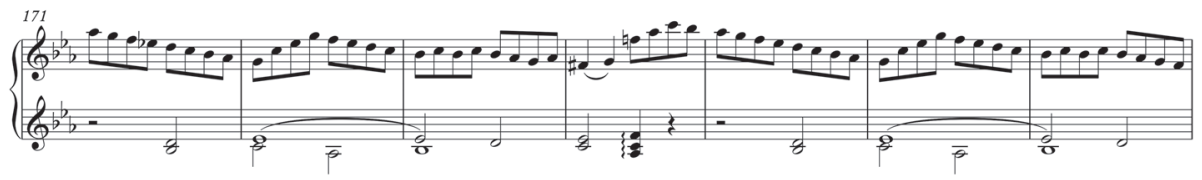




\section{EXEMPLE 4 (Continuation)}

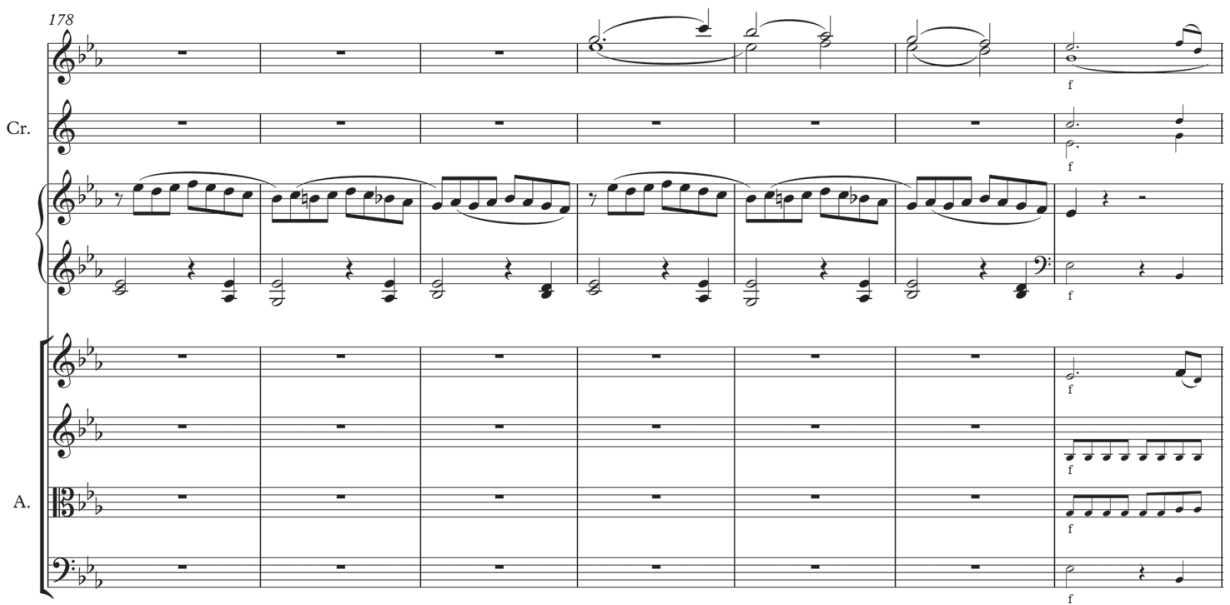

blanche = environ 120 à plus de 200 bpm d'une part, et entre blanche $=$ environ 80 à 105 bpm d'autre part. ${ }^{55}$ Par ailleurs, les changements de direction plus fréquents dans le graphique qui se rapporte au Concerto en Mi bémol confirment le caractère plus « heurté » de l'interprétation. Ce graphe présente par ailleurs un creux très marqué pour le $1^{\mathrm{er}}$ temps de la mes. 154. Ce creux est dû à une contrainte que

Tableau 2 Fluctuations de tempo dans le Concerto pour piano $n^{\circ} 9$ en Mi bémol majeur, K. 271, Presto, mes. 150-184

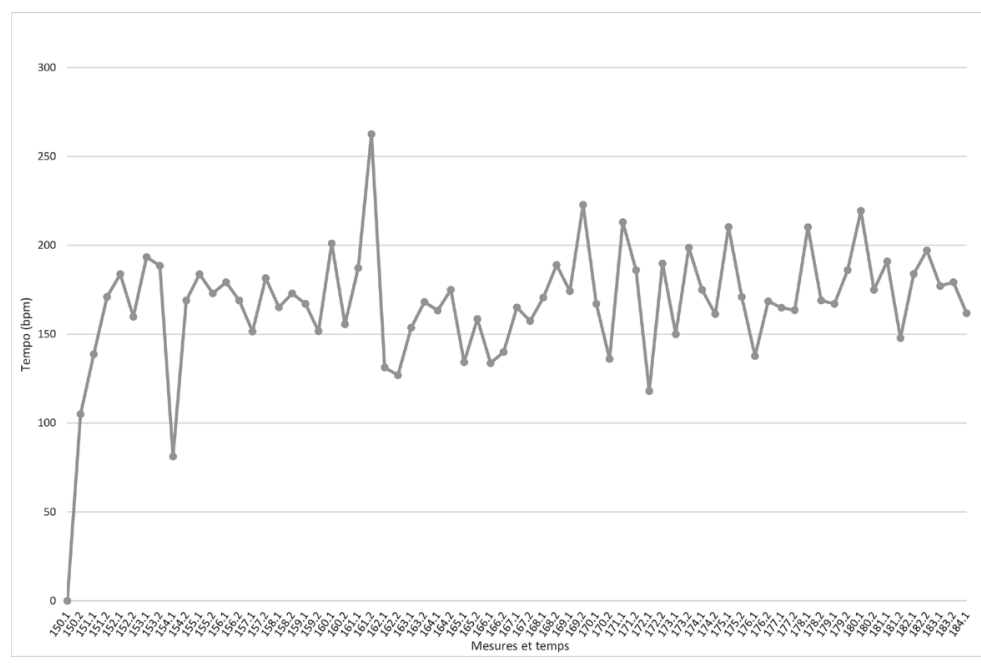

55. Les différences sont telles que le biais dû à la comparaison chiffres à chiffres de «zones » de tempo distinctes n'est pas significatif. Le biais provient du fait que plus le tempo est rapide, moins l'incrémentation du tempo a d'effet (les métronomes tiennent compte du phénomène). 
Tableau 3 Fluctuations de tempo dans le Concerto pour piano $n^{\circ} 17$ en Sol majeur, K. 453, Allegretto, mes. 17-32

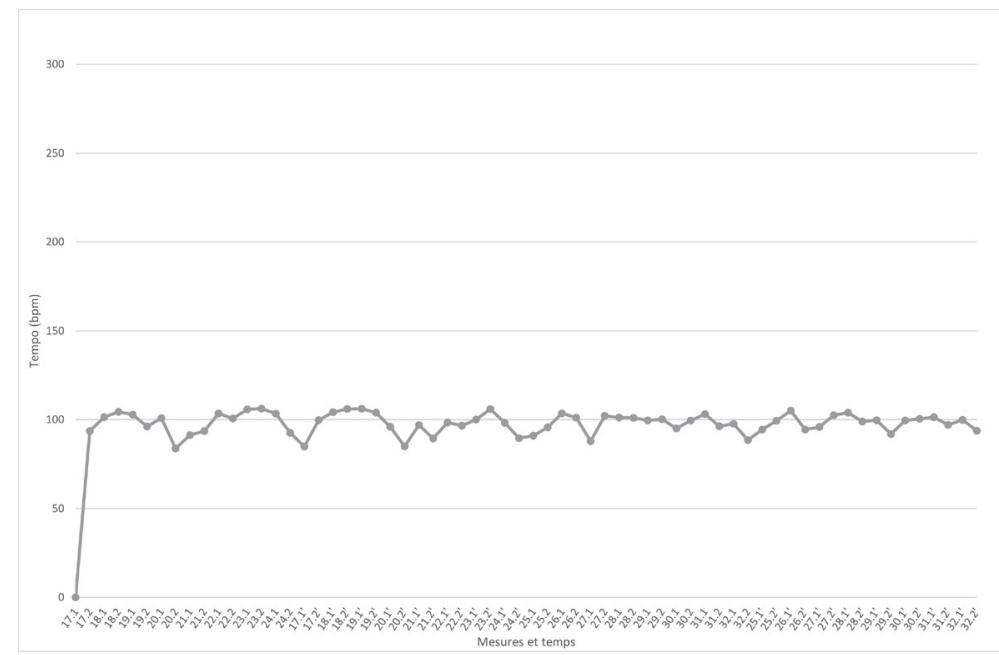

je me suis imposée lors de l'analyse pour répondre à un problème sur l'enregistrement. En effet, aux mes. 152-153, un flou dû soit au pianiste soit à la captation, a comme conséquence leur allongement. ${ }^{56} \mathrm{~J}$ 'ai choisi de ne pas ajouter de temps supplémentaire et d'attendre que la musique « retombe sur ses pieds».

La comparaison entre les orchestres, elle, fait peu de sens, dans la mesure où, dans les enregistrements tardifs, Dohnányi est accompagné par un ensemble instrumental qui n'atteint pas un standard technique élevé. C'est bien normal, cet orchestre est composé, pour rappel, d'étudiants.

Que retenir de ce bref parcours ? Peut-on véritablement comparer ces interprétations au vu de leur inscription chronologique éloignée ? si oui, que penser de ces ressemblances ? Dohnányi joue-t-il, malgré ses déclarations, de façon globalement similaire Mozart en studio et en concert ? ou alors, le « style de studio », devenu « mainstream », a-t-il déteint sur le «style de concert»? Une étude plus vaste de l'interprétation des œuvres pour piano de Mozart couvrant toute la période devrait contribuer à clarifier les choses. entre la fin du solo et l'entrée de l'orchestre. 


\section{Place des concertos pour piano de Mozart dans l'industrie du disque jusqu'en 1950}

Dix ans après l'enregistrement Columbia du Concerto en Sol, tous les « grands » concertos de Mozart, à savoir les $n^{\circ} 14-27$, sont sur le marché du disque, à l'exception du Concerto $n^{\circ} 16$ en Ré majeur, K. 451. Les informations détaillées à ce propose figurent dans le Tableau 4, qui répertorie les premières réalisations discographiques des concertos pour piano du compositeur jusqu'en $1950 .{ }^{57}$ Les pianistes qui jouent ces « grands » concertos sont dans l'ordre chronologique Georges Boskoff, Magda Tagliaferro, Arthur Rubinstein, Edwin Fischer, Artur Schnabel, Kathleen Long, Elly Ney, Walter Gieseking, Robert Casadesus et Lili Kraus. Les disques sortent principalement chez His Master's Voice, Columbia et Decca. Se constitue ainsi un sous-groupe dans l'œuvre de Mozart au niveau discographique. Beaucoup de ces enregistrements ont dû être bien diffusés. En effet, bon nombre d'archives sonores européennes et américaines conservent ces disques, et souvent en plusieurs exemplaires. Ils sont par ailleurs régulièrement disponibles sur le marché actuel du 78 tours.

L'étude reste à faire sur l'impact réel de ces réalisations sur la réception du genre du concerto pour piano mozartien de façon plus générale. Mais c'est durant ces années que ce groupe d'œuvres s'est véritablement installé dans le répertoire. La lecture de quelques textes en amont et en aval de la période en atteste. Dans son essai de 1903 intitulé The Classical Concerto, Donald Francis Tovey constate qu'il existe moins de grandes œuvres dans la vraie forme du concerto, à savoir pour lui une forme à la fois dramatique et poétique, que de grandes symphonies, " and of this small collection a good two-thirds has been contributed by Mozart, whose work has for the last fifty years been treated with neglect and lack of intelligent observation [...]. ${ }^{58}$ Lucien Chevaillier, dans La Revue musicale en 1911, constate lui aussi la rareté des concertos pour piano de Mozart au concert : «On rencontre aujourd'hui peu de programmes de concerts qui ne contiennent au moins une œuvre de Bach ou de Beethoven : en revanche, il est rare d'en trouver un où puisse se lire le nom de... Mozart. [...] Et c'est en ce moment aux concertos pour piano que je pense : l'œuvre la plus négligée, je dirais presque l'œuvre

57. J'ai constitué cette liste en croisant les informations des discographies scientifiques courantes avec celles des catalogues des grandes archives sonores européennes et nord-américaines. Quelques concertos sont réenregistrés par d'autres interprètes durant ces années. C'est notamment le cas du Concerto en Sol, joué par Edwin Fischer et son orchestre de chambre, qu'il dirige lui-même. La captation date de 1937 et est parue chez His Master's Voice (DB 3362-4).

58. Donald Francis Tovey, «The Classical Concerto » (1903), in Essays in Musical Analysis, vol. 3 Concertos (Londres : Oxford University Press, 1936). Réédité in Concertos and Choral Works. Selections from Essays in Musical Analysis (Mineola, New York : Dover, 2015), 3. 
TABleaU 4 Premiers enregistrements gramophoniques des concertos pour piano de Mozart jusqu'en 1950 (Les captations subséquentes des mêmes œuvres ne sont pas mentionnées)

\begin{tabular}{|c|c|c|c|c|}
\hline $\begin{array}{l}\text { Année } \\
\text { d'enregis- } \\
\text { trement }\end{array}$ & Euvre & Pianiste & Orchestre et chef & Label \\
\hline 1919 & $\begin{array}{l}\text { Concerto } n^{\circ} 9 \text { en } \mathrm{Mi} \\
\text { bémol majeur, K. } 271 \\
\text { (vraisemblablement), } \\
\text { Deuxième } \\
\text { mouvement, } \\
\text { arr. Busoni } \\
\end{array}$ & Busoni Ferruccio & - & $\begin{array}{l}\text { Columbia, } \\
\text { non publié }\end{array}$ \\
\hline 1928 & $\begin{array}{l}\text { Concerto } n^{\circ} 17 \text { en Sol } \\
\text { majeur, K. } 453\end{array}$ & $\begin{array}{l}\text { Dohnányi Ernst } \\
\text { von }\end{array}$ & $\begin{array}{l}\text { Budapest Philharmonic } \\
\text { Orchestra, } \\
\text { Ernst von Dohnányi }\end{array}$ & Columbia \\
\hline 1928 & $\begin{array}{l}\text { Mozart/Busoni, } \\
\text { Duettino concertante } \\
\text { [arr. de K. } \\
\text { 459, Troisième } \\
\text { mouvement, pour } \\
\text { piano à } 4 \text { mains] }\end{array}$ & $\begin{array}{l}\text { Vianna da Motta } \\
\text { José, } \\
\text { Mlle De Castello } \\
\text { Lopes }\end{array}$ & - & Pathé-Art \\
\hline 1930 & $\begin{array}{l}\text { Concerto } n^{\circ} 19 \text { en } \mathrm{Fa} \\
\text { majeur, K. } 459\end{array}$ & Boskoff Georges & $\begin{array}{l}\text { Orchestre Philharmonique } \\
\text { de Paris, } \\
\text { Gustave Cloëz }\end{array}$ & Columbia \\
\hline $1930-1931$ & $\begin{array}{l}\text { Concerto } n^{\circ} 26 \text { en Ré } \\
\text { majeur, K. } 537\end{array}$ & $\begin{array}{l}\text { Tagliaferro } \\
\text { Magda }\end{array}$ & $\begin{array}{l}\text { Orchestre Pasdeloup, } \\
\text { Reynaldo Hahn }\end{array}$ & Decca \\
\hline 1931 & $\begin{array}{l}\text { Concerto } n^{\circ} 23 \text { en La } \\
\text { majeur, K. } 488\end{array}$ & $\begin{array}{l}\text { Rubinstein } \\
\text { Arthur }\end{array}$ & $\begin{array}{l}\text { London Symphony } \\
\text { Orchestra, } \\
\text { John Barbirolli }\end{array}$ & $\begin{array}{l}\text { His } \\
\text { Master's } \\
\text { Voice }\end{array}$ \\
\hline 1931 & $\begin{array}{l}\text { Concerto } n^{\circ} 27 \text { en } \mathrm{Si} \\
\text { bémol majeur, } \\
\text { K. } 595\end{array}$ & $\begin{array}{l}\text { Casadesus } \\
\text { Robert }\end{array}$ & $\begin{array}{l}\text { ?, } \\
\text { Walter Straram }\end{array}$ & $\begin{array}{l}\text { Columbia, } \\
\text { non publié }\end{array}$ \\
\hline 1932 & $\begin{array}{l}\text { Concerto } n^{\circ} 20 \text { en ré } \\
\text { mineur, K. } 466\end{array}$ & Fischer Edwin & $\begin{array}{l}\text { Berliner Philharmoniker, } \\
\text { Eugen Jochum }\end{array}$ & $\begin{array}{l}\text { His } \\
\text { Master's } \\
\text { Voice, } \\
\text { non publié }\end{array}$ \\
\hline 1933 & $\begin{array}{l}\text { Concerto en Ré } \\
\text { majeur, K. } 107, \mathrm{n}^{\circ} 1\end{array}$ & $\begin{array}{l}\text { M. Mendler- } \\
\text { Schramm } \\
\text { (clavecin) }\end{array}$ & $\begin{array}{l}\text { Orchestre de chambre, } \\
?\end{array}$ & $\begin{array}{l}\text { His } \\
\text { Master's } \\
\text { Voice }\end{array}$ \\
\hline 1933 & $\begin{array}{l}\text { Concerto } n^{\circ} 20 \text { en ré } \\
\text { mineur, K. } 466\end{array}$ & Fischer Edwin & $\begin{array}{l}\text { London Philharmonic } \\
\text { Orchestra, } \\
\text { Edwin Fischer }\end{array}$ & $\begin{array}{l}\text { His } \\
\text { Master's } \\
\text { Voice }\end{array}$ \\
\hline 1934 & $\begin{array}{l}\text { Concerto } n^{\circ} 27 \text { en } \mathrm{Si} \\
\text { bémol majeur, K. } 595\end{array}$ & Schnabel Artur & $\begin{array}{l}\text { London Symphony } \\
\text { Orchestra, } \\
\text { John Barbirolli }\end{array}$ & $\begin{array}{l}\text { His } \\
\text { Master's } \\
\text { Voice }\end{array}$ \\
\hline 1935 & $\begin{array}{l}\text { Concerto } n^{\circ} 12 \text { en La } \\
\text { majeur, K. } 414\end{array}$ & Long Kathleen & $\begin{array}{l}\text { Boyd Neel Orchestra, } \\
\text { Boyd Neel }\end{array}$ & Decca \\
\hline
\end{tabular}


TABLEaU 4 (Continuation)

\begin{tabular}{|c|c|c|c|c|}
\hline $\begin{array}{l}\text { Année } \\
\text { d'enregis- } \\
\text { trement }\end{array}$ & Euvre & Pianiste & Orchestre et chef & Label \\
\hline 1935 & $\begin{array}{l}\text { Concerto } n^{\circ} 14 \text { en } M i \\
\text { bémol majeur, K. } 449\end{array}$ & Long Kathleen & $\begin{array}{l}\text { Boyd Neel Orchestra } \\
\text { Boyd Neel }\end{array}$ & Decca \\
\hline 1935 & $\begin{array}{l}\text { Concerto } n^{\circ} 15 \text { en } \mathrm{Si} \\
\text { bémol majeur, K. } 450\end{array}$ & Ney Elly & $\begin{array}{l}\text { Kammerorchester, } \\
\text { Willem van Hoogstraaten }\end{array}$ & Electrola \\
\hline 1935 & $\begin{array}{l}\text { Concerto } n^{\circ} 22 \text { en } \mathrm{Mi} \\
\text { bémol majeur, K. } 482\end{array}$ & Fischer Edwin & $\begin{array}{l}\text { John Barbirolli Chamber } \\
\text { Orchestra, } \\
\text { John Barbirolli }\end{array}$ & $\begin{array}{l}\text { His } \\
\text { Master's } \\
\text { Voice }\end{array}$ \\
\hline 1936 & $\begin{array}{l}\text { Concerto } n^{\circ} 1 \text { en } \mathrm{Fa} \\
\text { majeur, K. } 37\end{array}$ & $\begin{array}{l}\text { Roesgen- } \\
\text { Champion } \\
\text { Marguerite } \\
\text { (clavecin) }\end{array}$ & $\begin{array}{l}\text { Orchestre Symphonique } \\
\text { de Paris } \\
\text { Marius-François Gaillard }\end{array}$ & Columbia \\
\hline 1936 & $\begin{array}{l}\text { Concerto } n^{\circ} 9 \text { en } \mathrm{Mi} \\
\text { bémol majeur, K. } 271\end{array}$ & Gieseking Walter & $\begin{array}{l}\text { Orchester der Staatsoper } \\
\text { Berlin, } \\
\text { Hans Rosbaud }\end{array}$ & Columbia \\
\hline 1936 & $\begin{array}{l}\text { Concerto } n^{\circ} 10 \text { pour } \\
\text { deux pianos en Mi } \\
\text { bémol majeur, K. } 365\end{array}$ & $\begin{array}{l}\text { Schnabel Artur, } \\
\text { Schnabel Karl } \\
\text { Ulrich }\end{array}$ & $\begin{array}{l}\text { London Symphony } \\
\text { Orchestra, } \\
\text { Adrian Boult }\end{array}$ & \begin{tabular}{|l} 
His \\
Master's \\
Voice \\
\end{tabular} \\
\hline 1937 & $\begin{array}{l}\text { Concerto } n^{\circ} 21 \text { en Do } \\
\text { majeur, K. } 467\end{array}$ & Schnabel Artur & $\begin{array}{l}\text { London Symphony } \\
\text { Orchestra, } \\
\text { Malcolm Sargent } \\
\end{array}$ & $\begin{array}{l}\text { His } \\
\text { Master's } \\
\text { Voice }\end{array}$ \\
\hline 1937 & $\begin{array}{l}\text { Concerto } n^{\circ} 24 \text { en do } \\
\text { mineur, K. } 491\end{array}$ & $\begin{array}{l}\text { Casadesus } \\
\text { Robert }\end{array}$ & $\begin{array}{l}\text { Orchestre Symphonique } \\
\text { de Paris, } \\
\text { Eugène Bigot }\end{array}$ & Columbia \\
\hline 1937 & $\begin{array}{l}\text { Concerto } n^{\circ} 24 \text { en do } \\
\text { mineur, K. } 491\end{array}$ & Fischer Edwin & $\begin{array}{l}\text { London Philharmonic } \\
\text { Orchestra, } \\
\text { Lawrance Collingwood }\end{array}$ & $\begin{array}{l}\text { His } \\
\text { Master's } \\
\text { Voice } \\
\end{array}$ \\
\hline 1938 & $\begin{array}{l}\text { Concerto } n^{\circ} 18 \text { en } \mathrm{Si} \\
\text { bémol majeur, K. } 456\end{array}$ & Kraus Lili & $\begin{array}{l}\text { London Philharmonic } \\
\text { Orchestra, } \\
\text { Walter Goehr }\end{array}$ & Parlophone \\
\hline 1938 & $\begin{array}{l}\text { Concerto } n^{\circ} 25 \text { en Do } \\
\text { majeur, K. } 503\end{array}$ & Long Kathleen & $\begin{array}{l}\text { Boyd Neel Orchestra, } \\
\text { Boyd Neel }\end{array}$ & Decca \\
\hline 1940 & $\begin{array}{l}\text { Concerto } n^{\circ} 4 \text { en Sol } \\
\text { majeur, K. } 41\end{array}$ & $\begin{array}{l}\text { Roesgen- } \\
\text { Champion } \\
\text { Marguerite } \\
\end{array}$ & $\begin{array}{l}\text { Orchestre Lamoureux, } \\
\text { Albert Wolff }\end{array}$ & Columbia \\
\hline 1949 & $\begin{array}{l}\text { Concerto } n^{\circ} 16 \text { en Ré } \\
\text { majeur, K. } 451 \\
\text { (incomplet) }\end{array}$ & Humby Betty & $\begin{array}{l}\text { Royal Philharmonic } \\
\text { Orchestra, } \\
\text { Sir Thomas Beecham }\end{array}$ & \begin{tabular}{|l} 
His \\
Master's \\
Voice, \\
non publié \\
\end{tabular} \\
\hline
\end{tabular}


méprisée [...]. $»^{59} \mathrm{Il}$ plaide ensuite pour ces derniers : «Je demande qu'on lise les concertos de Mozart ; je demande aussi qu'on les joue. $»^{60}$

Or en 1939, Cuthbert Morton Girdlestone, constate dans son livre précisément sur les concertos pour piano de Mozart « l'enthousiasme grandissant de notre époque pour les concertos de Mozart [...]. » ${ }^{61}$ De même, neuf ans plus tard, Arthur Hutchings peut écrire que " gone are the days, let us hope, when a Mozart concerto was considered fit pabulum for the year's best girl preparing for a terminal concert. $»^{62}$ Cette affirmation du genre concerto s'accompagne d'un repositionnement, toujours valide aujourd'hui, dans la hiérarchie de la production mozartienne. Ainsi, par exemple, W. McNaught relève la même année dans The Musical Times : «Between the wars Mozart's music took new life in our concert halls. Musicians, performers, concert-givers and the listening and reading audience found that Mozart was vital, indispensable and endless; and by mutual encouragement a large inner ring of our musical community came to believe that it was genuinely in touch with the composer. A special cause of satisfaction lay in the discovery of the piano concertos, not as a work in D minor [K. 466] and another one in A [probablement K. 488], but as a body of masterpieces that lived apart from the rest of music and formed the most intimate revelation of the composer's. ${ }^{63}$ Alfred Einstein ne dit pas autre chose dans son importante biographie, parue d'abord en anglais en 1945 : «C'est au fond dans le concerto pour piano que sa création [celle de Mozart] revêt sa forme la plus foncièrement originale [...]. » ${ }^{64}$

59. Lucien Chevaillier, « Pour Mozart. Les concertos de piano », La Revue musicale 11/10 (novembre 1911), 222.

60. Ibid., 231.

61. Cuthbert Morton Girdlestone, W.-A. Mozart et ses concertos pour piano, 2 tomes (Paris : Librairie Fischbacher, 1939), 513.

62. Arthur Hutchings, A Companion to Mozart's Piano Concertos (Londres : Oxford University Press, 1948), 199.

63. W. McN[aught], « Mozart's Piano Concertos », The Musical Times 89/1263 (May 1948), 137.

64. Alfred Einstein, Mozart : l'homme et l'œuvre, trad. Jacques Delalande (Paris : Gallimard, 1991 [en anglais : $\left.\left.{ }^{1} 1945\right]\right), 366$. (Tel, 175). 